\title{
Critical Sublattice Symmetry Breaking: A Universal Criterion for Dirac Cone Splitting
}

\author{
Ritesh Kumar $^{\dagger}$, Deya Das ${ }^{\dagger}$, Enrique Muñoz ${ }^{\ddagger}$ and Abhishek K. Singh ${ }^{\dagger *}$ \\ $\dagger$ Materials Research Centre, Indian Institute of Science, Bangalore 560012, India \\ †Facultad de Física, Pontificia Universidad Católica de Chile, Vicuña Mackenna 4860 \\ Santiago, Chile
}

\section{Analytical theory}

In this section, we present a theory for Dirac cone splitting in graphene van der Waals heterostructures, where the substrate may induce chiral symmetry breaking. Our model is based on a tight-binding representation of single-layer graphene, subjected to an on-site potential representing the proximity of the substrate atoms. The fact that this local potential may differ at each site, provides a mechanism for chiral symmetry breaking and the corresponding splitting of the Dirac cone in graphene. From an effective tight-binding model for the graphene-heterostructure, a simple stoichiometric rule is obtained to predict the conditions for Dirac cone splitting in the energy spectrum.

\subsection{The tight-binding free Hamiltonian for single-layer graphene}

The point group of the honeycomb lattice that describes the single-layer graphene is $C_{6 v}$. It can be decomposed as $C_{6 v}=C_{3 v} \otimes \mathbf{Z}_{2}$, i.e. as the product of the point group of the triangular lattice and the $\mathbf{Z}_{2}$ sublattice symmetry group. We denote the sites at each traingular sublattice A, B as

$$
\mathbf{R}_{A}=n_{1} \mathbf{a}_{1}+n_{2} \mathbf{a}_{2}, \quad \mathbf{R}_{B}=\mathbf{R}_{A}+\mathbf{d}_{j} .
$$

Here, we have defined the set of basis vectors

$$
\begin{aligned}
& \mathbf{a}_{1}=\sqrt{3} a(1,0), \quad \mathbf{a}_{2}=\frac{\sqrt{3}}{2} a(1, \sqrt{3}) \\
& \mathbf{d}_{1}=\frac{a}{2}(\sqrt{3}, 1), \quad \mathbf{d}_{2}=\frac{a}{2}(-\sqrt{3}, 1), \quad \mathbf{d}_{3}=-a(0,1) .
\end{aligned}
$$


Table S1: Summary of graphene heterostructures

\begin{tabular}{|c|c|c|c|c|c|}
\hline 2D substrate & $\begin{array}{l}\text { Lattice mis- } \\
\text { match }(\%)\end{array}$ & $\begin{array}{l}\text { Inequivalent }(A, B) \\
\text { pairs }\end{array}$ & $\begin{array}{l}\text { Equivalent }(A, B) \\
\text { pairs }\end{array}$ & $\begin{array}{l}\text { Splitting in Dirac } \\
\text { cone }(\mathrm{meV})\end{array}$ & $\begin{array}{ll}\text { Binding } & \text { energy } \\
(\mathrm{meV} / \AA) & \end{array}$ \\
\hline h-BN $(\mathrm{AA})^{\dagger}$ & 1.59 & 1 & 0 & 76 & -9.62 \\
\hline$h-B N(A B)^{\dagger}$ & 1.59 & 1 & 0 & 51 & -13.94 \\
\hline $\mathrm{C}_{3} \mathrm{~N}(\mathrm{AA})$ & 1.57 & 6 & 6 & 00 & -13.40 \\
\hline $\mathrm{C}_{3} \mathrm{~N}(\mathrm{AB})$ & 1.57 & 12 & 0 & 74 & -13.60 \\
\hline $\mathrm{C}_{3} \mathrm{~N}_{3}$ (AA) & 3.51 & 18 & 9 & 190 & 19.90 \\
\hline $\mathrm{C}_{3} \mathrm{~N}_{3}(\mathrm{AB})$ & 3.51 & 24 & 3 & 160 & 17.48 \\
\hline$h-\mathrm{C}_{3} \mathrm{~N}_{4}(\mathrm{AA})^{\dagger}$ & 3.78 & 27 & 0 & 31 & 19.00 \\
\hline$h-\mathrm{C}_{3} \mathrm{~N}_{4}(\mathrm{AB})^{\dagger}$ & 3.78 & 27 & 0 & 52 & 17.69 \\
\hline$t-\mathrm{C}_{3} \mathrm{~N}_{4}(\mathrm{AA})^{\dagger}$ & 3.00 & 12 & 0 & 44 & -0.54 \\
\hline$t-\mathrm{C}_{3} \mathrm{~N}_{4}(\mathrm{AB})^{\dagger}$ & 3.00 & 12 & 0 & 32 & -1.17 \\
\hline$h-\mathrm{C}_{4} \mathrm{~N}_{3}(\mathrm{AA})$ & 2.08 & 27 & 0 & 124 & -9.74 \\
\hline$h-\mathrm{C}_{4} \mathrm{~N}_{3}(\mathrm{AB})$ & 2.08 & 27 & 0 & 36 & -12.87 \\
\hline$t-\mathrm{C}_{4} \mathrm{~N}_{3}(\mathrm{AA})$ & 3.13 & 12 & 0 & 87 & -3.50 \\
\hline$t-\mathrm{C}_{4} \mathrm{~N}_{3}(\mathrm{AB})$ & 3.13 & 12 & 0 & 29 & -6.18 \\
\hline $\mathrm{BC}_{3}(\mathrm{AA})$ & 4.54 & 6 & 6 & 00 & 32.00 \\
\hline $\mathrm{BC}_{3}(\mathrm{AB})^{\dagger}$ & 4.54 & 12 & 0 & 74 & 30.66 \\
\hline $\mathrm{PtS}_{2}$ & 3.54 & 13 & 14 & 00 & -2.20 \\
\hline $\mathrm{PtSe}_{2}(1)$ & 1.30 & 9 & 18 & 00 & -10.56 \\
\hline $\mathrm{PtSe}_{2}(2)$ & 1.30 & 18 & 9 & 18 & -10.55 \\
\hline $\mathrm{MoS}_{2}$ & 3.24 & 18 & 30 & 00 & -11.32 \\
\hline $\mathrm{MoSe}_{2}$ & 0.80 & 16 & 32 & 00 & -16.99 \\
\hline $\mathrm{MoTe}_{2}$ & 4.37 & 12 & 15 & 00 & 36.57 \\
\hline $\mathrm{SnS}_{2}$ & 0.13 & 3 & 24 & 00 & -14.45 \\
\hline $\mathrm{WS}_{2}$ & 3.24 & 21 & 27 & 00 & 6.91 \\
\hline $\mathrm{HfO}_{2}$ & 1.52 & 18 & 30 & 00 & -15.26 \\
\hline $\mathrm{Ti}_{2} \mathrm{CO}_{2}$ & 2.27 & 11 & 28 & 00 & -0.41 \\
\hline Phosphorene & 0.80 & 2 & 22 & 00 & 1.01 \\
\hline
\end{tabular}


(a)

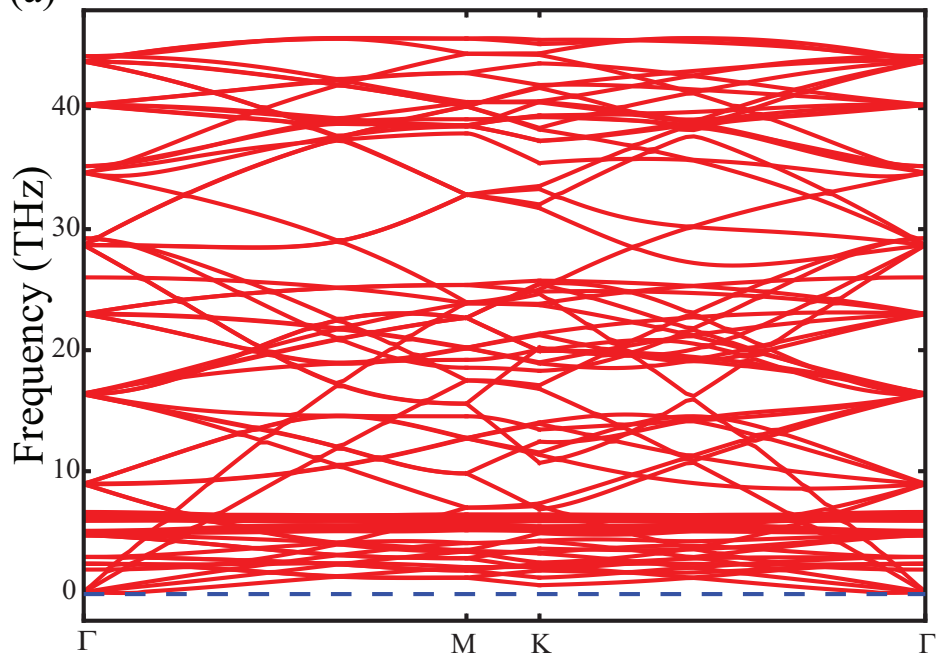

(b)

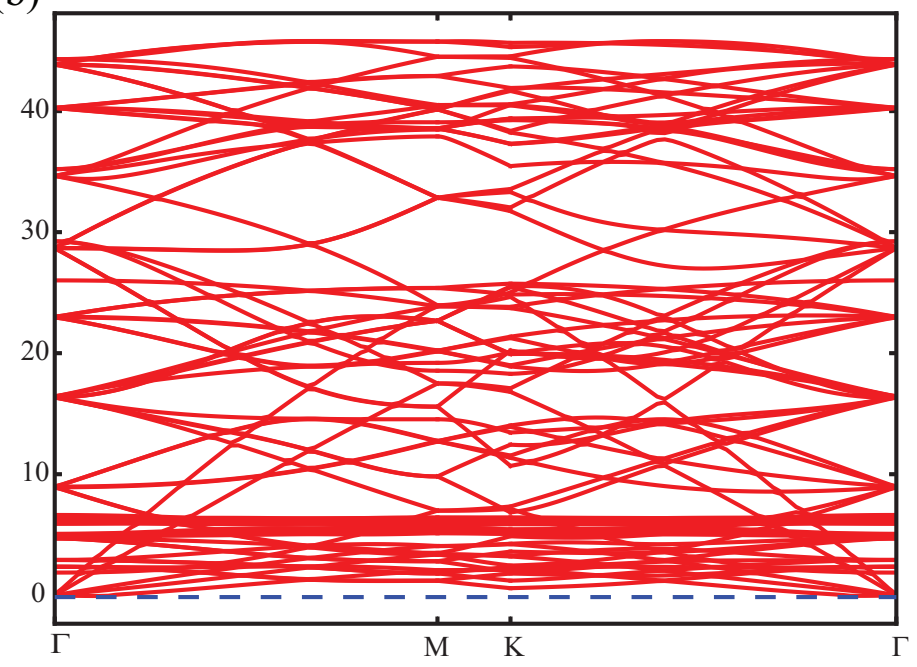

Figure S1: Phonon band structures of (a) stacking 1 and (b) stacking 2 of gr/PtSe $\mathrm{P}_{2}$ heterostructures.

(a)

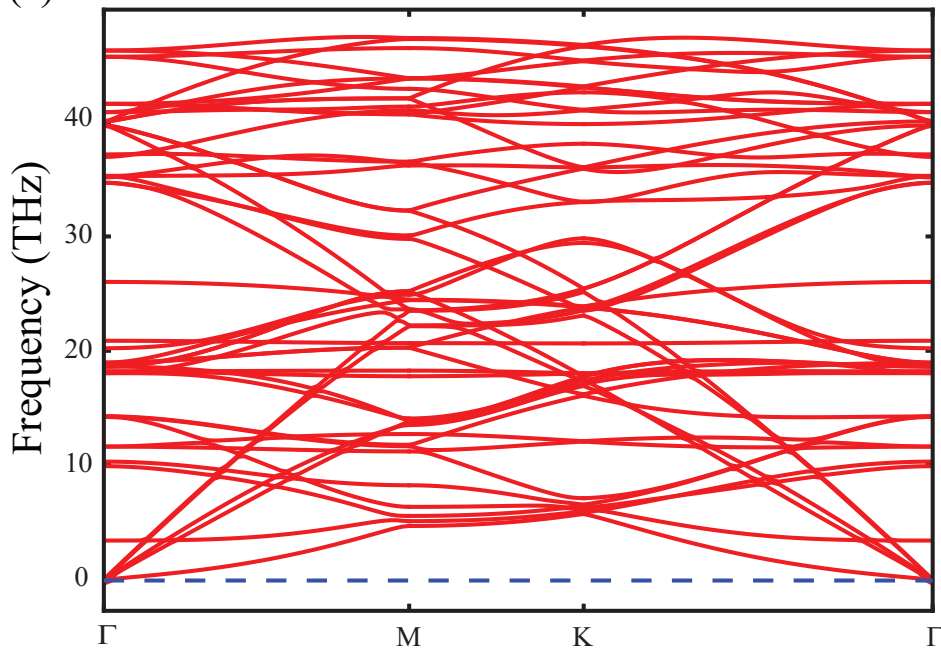

(b)

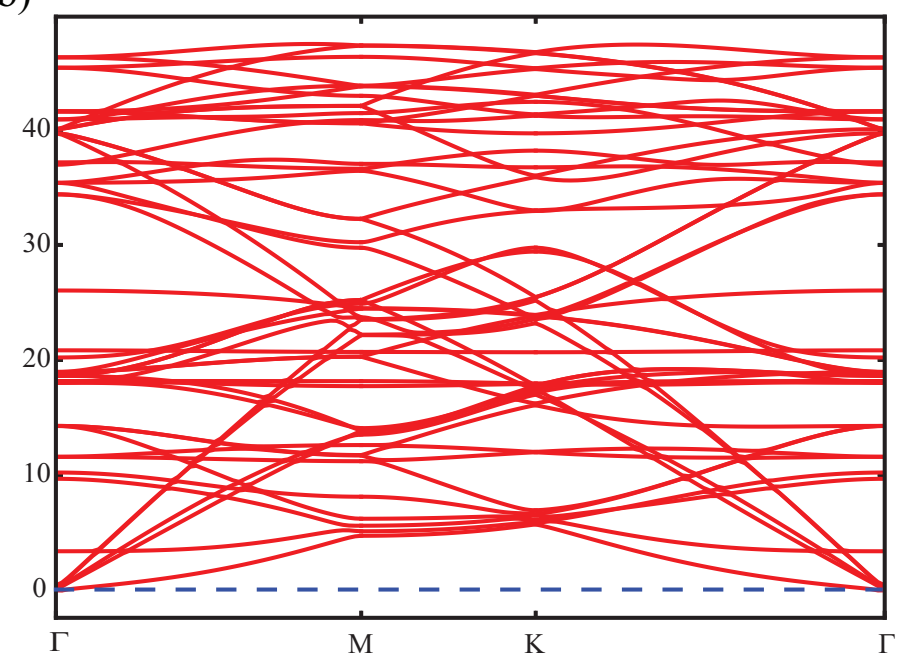

Figure S2: Phonon band structures of (a) AA and (b) $\mathrm{AB}$ stacking of $\mathrm{gr} / \mathrm{C}_{3} \mathrm{~N}$ heterostructures. 
(a)

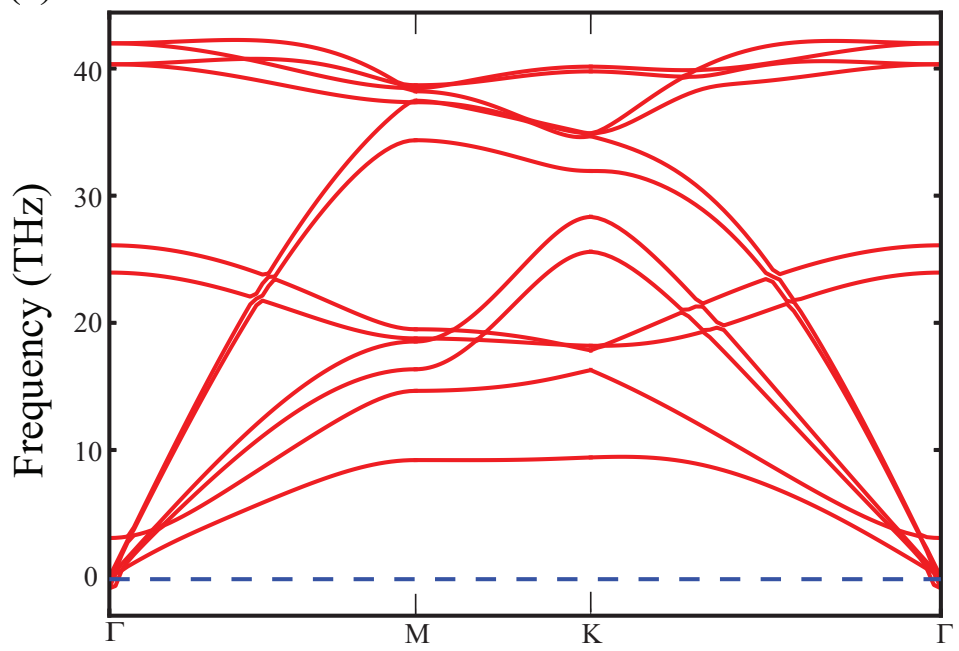

(b)

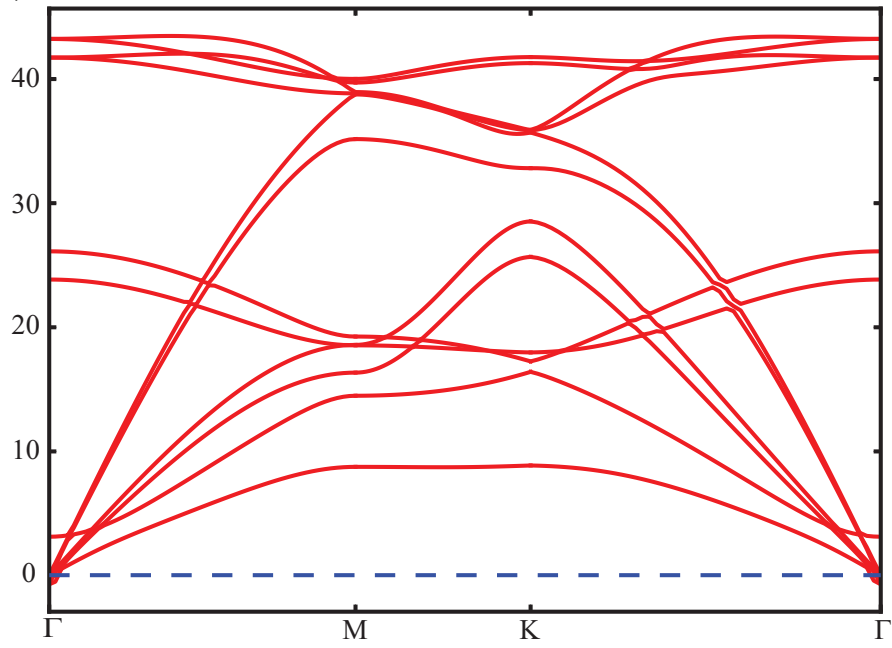

Figure S3: Phonon band structures of (a) AA and (b) AB stacking of gr/h-BN heterostructures.

(a)

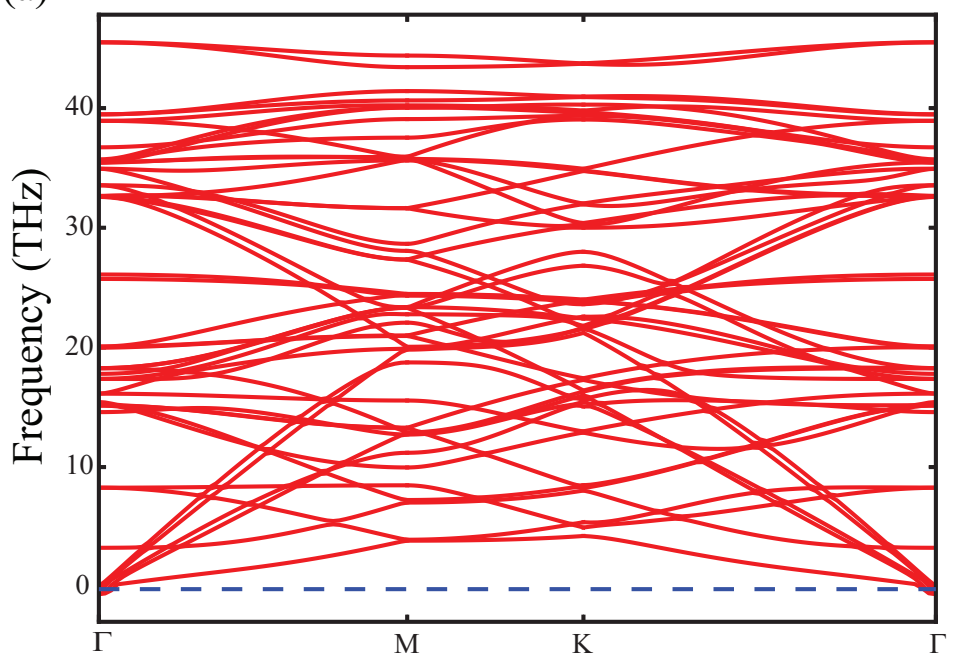

(b)

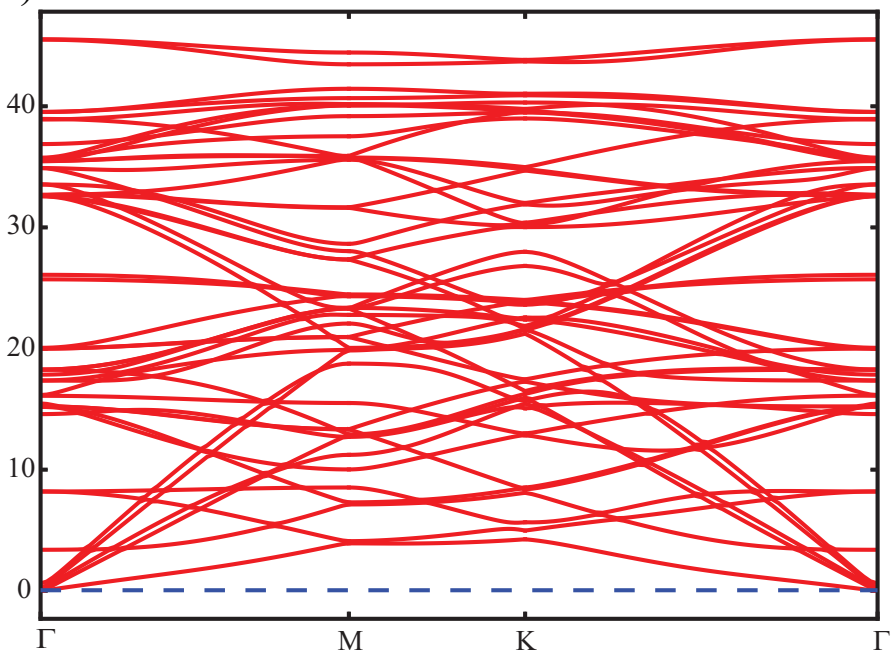

Figure S4: Phonon band structures of (a) $\mathrm{AA}$ and (b) $\mathrm{AB}$ stacking of $\mathrm{gr} / \mathrm{BC}_{3}$ heterostructures. 
The tight-binding Hamiltonian for single-layer graphene, in the absence of external potentials and interactions, is given by the operator

$$
\hat{H}_{0}=-t \sum_{\mathbf{R}_{A}, j, \sigma}\left(\hat{a}_{\sigma}^{\dagger}\left(\mathbf{R}_{A}\right) \hat{b}_{\sigma}\left(\mathbf{R}_{A}+\mathbf{d}_{j}\right)+\text { h.c. }\right) .
$$

Here, we have defined fermionic creation/annihilation operators $\hat{a}_{\sigma}\left(\mathbf{R}_{A}\right)$ and $\hat{b}_{\sigma}\left(\mathbf{R}_{B}\right)$ at each sublattice $(\mathrm{A}, \mathrm{B})$, in the Wannier (position) representation, while $\sigma= \pm 1 / 2$ represents the two states for electronic spin. The operators in the Wannier representation are connected to the Bloch (crystal momentum) representation by the series expansion

$$
\begin{aligned}
\hat{a}_{\sigma}\left(\mathbf{R}_{A}\right) & =\frac{1}{\sqrt{N}} \sum_{\mathbf{k}} e^{-i \mathbf{k} \cdot \mathbf{R}_{A}} \hat{a}_{\sigma, \mathbf{k}} \\
\hat{b}_{\sigma}\left(\mathbf{R}_{B}\right) & =\frac{1}{\sqrt{N}} \sum_{\mathbf{k}} e^{-i \mathbf{k} \cdot \mathbf{R}_{B}} \hat{b}_{\sigma, \mathbf{k}}
\end{aligned}
$$

with $N$ the total number of atoms in each sublattice (i.e. $N_{A}=N_{B}=N$ ). Substituting Eq.(S4) into Eq.(S3), and applying the identity $N^{-1} \sum_{\mathbf{R}_{A}} e^{-i\left(\mathbf{k}-\mathbf{k}^{\prime}\right) \cdot \mathbf{R}_{A}}=\delta_{\mathbf{k}, \mathbf{k}^{\prime}}$, we obtain the representation of the free Hamiltonian in crystal momentum space

$$
\hat{H}_{0}=\sum_{\mathbf{k}, \sigma}\left(\hat{a}_{\sigma, \mathbf{k}}^{\dagger} \hat{b}_{\sigma, \mathbf{k}}^{\dagger}\right)\left(\begin{array}{cc}
0 & -\gamma_{\mathbf{k}}^{*} \\
-\gamma_{\mathbf{k}} & 0
\end{array}\right)\left(\begin{array}{c}
\hat{a}_{\sigma, \mathbf{k}} \\
\hat{b}_{\sigma, \mathbf{k}}
\end{array}\right)
$$

Here, we have defined the parameter

$$
\gamma_{\mathbf{k}}=t \sum_{j=1}^{3} e^{i \mathbf{k} \cdot \mathbf{d}_{j}}
$$

The exact energy eigenvalues of the Hamiltonian Eq.(S5) are given by

$$
E_{ \pm}(\mathbf{k})= \pm\left|\gamma_{\mathbf{k}}\right|= \pm t \sqrt{1+4 \cos ^{2}\left(\sqrt{3} a k_{x} / 2\right)\left[1+\cos \left(3 a k_{y} / 2\right)\right]}
$$

The Dirac points are identified from Eqs.(S6-S7), by imposing the condition

$$
\gamma_{\mathbf{k}=\mathbf{K}_{D}^{ \pm}}=0 \Longrightarrow \mathbf{K}_{D}^{ \pm}= \pm \frac{4 \pi}{3 \sqrt{3} a}(1,0)
$$

The Dirac effective Hamiltonian is obtained by expanding the parameters $\gamma_{\mathbf{k}}$ in the vicinity of each Dirac point, i.e. at $\mathbf{k}=\mathbf{K}_{D}^{ \pm}+\mathbf{q}$ :

$$
\gamma_{\mathbf{q}}^{ \pm} \equiv \gamma_{\mathbf{k}=\mathbf{K}_{D}^{ \pm}+\mathbf{q}}=t \sum_{j=1}^{3} e^{i\left(\mathbf{K}_{D}^{ \pm}+\mathbf{q}\right) \cdot \mathbf{d}_{j}} \sim t \sum_{j=1}^{3} e^{i \mathbf{K}_{D}^{ \pm} \cdot \mathbf{d}_{j}}\left(1+i \mathbf{q} \cdot \mathbf{d}_{j}\right)=\mp \frac{3}{2} t a\left(q_{x} \pm i q_{y}\right)
$$


We define the Fermi velocity by $\hbar v_{F}=\frac{3}{2} t a$, as well as the spinor operators in the vicinity of each valley $\mathbf{K}_{D}^{ \pm}$as follows

$$
\hat{\psi}_{\sigma, \mathbf{q}}^{(+)}=\left(\begin{array}{c}
\hat{a}_{\sigma, \mathbf{K}_{D}^{+}+\mathbf{q}} \\
\hat{b}_{\sigma, \mathbf{K}_{D}^{+}+\mathbf{q}}
\end{array}\right), \hat{\psi}_{\sigma, \mathbf{q}}^{(-)}=\left(\begin{array}{c}
\hat{b}_{\sigma, \mathbf{K}_{D}^{-}+\mathbf{q}} \\
\hat{a}_{\sigma, \mathbf{K}_{D}^{-}+\mathbf{q}}^{-}
\end{array}\right) .
$$

The full non-interacting Hamiltonian, including the contribution of both valleys, is thus given by the expression

$$
\hat{H}_{0}=\hbar v_{F} \sum_{\mathbf{k}, \sigma} \hat{\Psi}_{\sigma}^{\dagger}(\mathbf{q})\left(\begin{array}{cc}
\vec{\sigma} \cdot \mathbf{q} & 0 \\
0 & -\vec{\sigma} \cdot \mathbf{q}
\end{array}\right) \hat{\Psi}_{\sigma}(\mathbf{q})
$$

where $\vec{\sigma}=\left(\hat{\sigma}_{x}, \hat{\sigma}_{y}\right)$ is a vector array of Pauli matrices,

$$
\hat{\sigma}_{x}=\left(\begin{array}{ll}
0 & 1 \\
1 & 0
\end{array}\right), \quad \hat{\sigma}_{y}=\left(\begin{array}{cc}
0 & -i \\
i & 0
\end{array}\right), \quad \hat{\sigma}_{z}=\left(\begin{array}{cc}
1 & 0 \\
0 & -1
\end{array}\right), \quad \hat{\sigma}_{0}=\left(\begin{array}{ll}
1 & 0 \\
0 & 1
\end{array}\right) .
$$

In Eq.(S10) we have also defined the 4-component spinor operator

$$
\hat{\Psi}_{\sigma}(\mathbf{q})=\left(\begin{array}{c}
\hat{\psi}_{\sigma, \mathbf{q}}^{(+)} \\
\hat{\psi}_{\sigma, \mathbf{q}}^{(-)}
\end{array}\right)
$$

\section{Chiral symmetry breaking due to the presence of a substrate}

In the graphene-based Van der Waals heterostructures, the presence of the other material in the vicinity of single-layer graphene modifies its spectral properties, and in particular under certain conditions is shown to induce a gap opening at the Dirac cone. One of the mechanisms that cause this gap opening is related to the alignment of the substrate with the graphene lattice, which can create an effective external potential that breaks the sublattice symmetry, and hence the chiral symmetry. In order to capture this effect, let us consider the following interaction Hamiltonian

$$
\hat{H}_{i n t}=\sum_{\sigma, \mathbf{R}_{A}} V\left(\mathbf{R}_{A}\right) \hat{a}_{\sigma}^{\dagger}\left(\mathbf{R}_{A}\right) \hat{a}_{\sigma}\left(\mathbf{R}_{A}\right)+\sum_{\sigma, \mathbf{R}_{B}} V\left(\mathbf{R}_{B}\right) \hat{b}_{\sigma}^{\dagger}\left(\mathbf{R}_{B}\right) \hat{b}_{\sigma}\left(\mathbf{R}_{B}\right),
$$

where, $V\left(\mathbf{R}_{A, B}\right)$ represents the effective local potential that acts at site $\mathbf{R}_{A, B}$ of the graphene lattice, due to the presence of the substrate. Using Eq.(S4), we can express the interaction Hamiltonian in momentum space

$$
\hat{H}_{i n t}=\sum_{\sigma, \mathbf{k}, \mathbf{k}^{\prime}} V^{(A)}\left(\mathbf{k}-\mathbf{k}^{\prime}\right) \hat{a}_{\sigma \mathbf{k}}^{\dagger} \hat{a}_{\sigma \mathbf{k}^{\prime}}+\sum_{\sigma, \mathbf{k}, \mathbf{k}^{\prime}} V^{(B)}\left(\mathbf{k}-\mathbf{k}^{\prime}\right) \hat{b}_{\sigma \mathbf{k}}^{\dagger} \hat{b}_{\sigma \mathbf{k}^{\prime}}
$$

where, we have defined the lattice-Fourier transforms of the local potential acting over sublattice sites $\mathbf{R}_{A}$ and $\mathbf{R}_{B}$, respectively, by the expressions

$$
\begin{aligned}
V^{(A)}\left(\mathbf{k}-\mathbf{k}^{\prime}\right) & =\frac{1}{N} \sum_{\mathbf{R}_{A}} e^{i\left(\mathbf{k}-\mathbf{k}^{\prime}\right) \cdot \mathbf{R}_{A}} V\left(\mathbf{R}_{A}\right), \\
V^{(B)}\left(\mathbf{k}-\mathbf{k}^{\prime}\right) & =\frac{1}{N} \sum_{\mathbf{R}_{B}} e^{i\left(\mathbf{k}-\mathbf{k}^{\prime}\right) \cdot \mathbf{R}_{B}} V\left(\mathbf{R}_{B}\right) .
\end{aligned}
$$


As illustrated in several examples along the main text of the paper, the presence of the substrate reduces the symmetry, along with expansion of the unit cell. Therefore, if $N$ is the total number of carbon atoms in the $A$ and $B$ sublattices (with one $n_{A}=n_{B}=1$ atom of each sublattice per unit cell), the redefinition of the enlarged unit cell containing $n_{A}=n_{B}=n$ will satisfy $N=n N_{c}$, with $N_{c}$ the total number of unit cells. Let us resort to a different set of coordinates, such that $\mathbf{R}_{c}$ represents the vector indicating the position of the center of each unit cell, for $c=1, \ldots, N_{c}$. With this definition, the position of each $A$ or $B$ atom in the original sublattice can be written as

$$
\mathbf{R}_{A}=\mathbf{R}_{c}+\mathbf{r}_{A}^{(j)}, \quad \mathbf{R}_{B}=\mathbf{R}_{c}+\mathbf{r}_{B}^{(j)},
$$

with $1 \leq j \leq n$, for $n$ the total number of atoms $A$ or $B$ within each unit cell, and $\mathbf{r}_{(A, B)}^{(j)}$ the position vector of each atom within the unit cell. Notice that this coordinate vectors are independent of the cell coordinates $\mathbf{R}_{c}$. The same applied to the values of the local potential induced by the presence of the substrate, whose values will depend only on the internal coordinates of the cell, i.e.

$$
\begin{aligned}
V\left(\mathbf{R}_{A}\right) & =V\left(\mathbf{R}_{c}+\mathbf{r}_{A}^{(j)}\right)=V\left(\mathbf{r}_{A}^{(j)}\right) \\
V\left(\mathbf{R}_{B}\right) & =V\left(\mathbf{R}_{c}+\mathbf{r}_{B}^{(j)}\right)=V\left(\mathbf{r}_{B}^{(j)}\right) .
\end{aligned}
$$

Substituting Eq.(S17) into Eq.(S15), and using $N=n N_{c}$, we have

$$
\begin{aligned}
V^{(A)}\left(\mathbf{k}-\mathbf{k}^{\prime}\right) & =\frac{1}{n N_{c}} \sum_{\mathbf{R}_{c}} \sum_{j=1}^{n} e^{i\left(\mathbf{k}-\mathbf{k}^{\prime}\right) \cdot\left(\mathbf{R}_{c}+\mathbf{r}_{A}^{(j)}\right)} V\left(\mathbf{R}_{c}+\mathbf{r}_{A}^{(j)}\right) \\
& =\left(\frac{1}{N_{c}} \sum_{\mathbf{R}_{c}} e^{i\left(\mathbf{k}-\mathbf{k}^{\prime}\right) \cdot \mathbf{R}_{c}}\right) \frac{1}{n} \sum_{j=1}^{n} e^{i\left(\mathbf{k}-\mathbf{k}^{\prime}\right) \cdot \mathbf{r}_{A}^{(j)}} V\left(\mathbf{r}_{A}^{(j)}\right) \\
& =\delta_{\mathbf{k}, \mathbf{k}^{\prime}} \frac{1}{n} \sum_{j=1}^{n} V\left(\mathbf{r}_{A}^{(j)}\right) \\
& =\delta_{\mathbf{k}, \mathbf{k}^{\prime}} V_{A} .
\end{aligned}
$$

Similarly, repeating the steps above we obtain

$$
V^{(B)}\left(\mathbf{k}-\mathbf{k}^{\prime}\right)=\delta_{\mathbf{k}, \mathbf{k}^{\prime}} V_{B},
$$

where we have defined the constants

$$
\begin{aligned}
V_{A} & =\frac{1}{n} \sum_{j=1}^{n} V\left(\mathbf{r}_{A}^{(j)}\right), \\
V_{B} & =\frac{1}{n} \sum_{j=1}^{n} V\left(\mathbf{r}_{B}^{(j)}\right) .
\end{aligned}
$$

Substituting Eq.(S18) and Eq.(S19) into Eq.(S14), the interaction Hamiltonian in lattice-Fourier space becomes

$$
\hat{H}_{i n t}=\sum_{\sigma, \mathbf{k}}\left[V_{A} \hat{a}_{\sigma \mathbf{k}}^{\dagger} \hat{a}_{\sigma \mathbf{k}}+V_{B} \hat{b}_{\sigma \mathbf{k}}^{\dagger} \hat{b}_{\sigma \mathbf{k}}\right]
$$




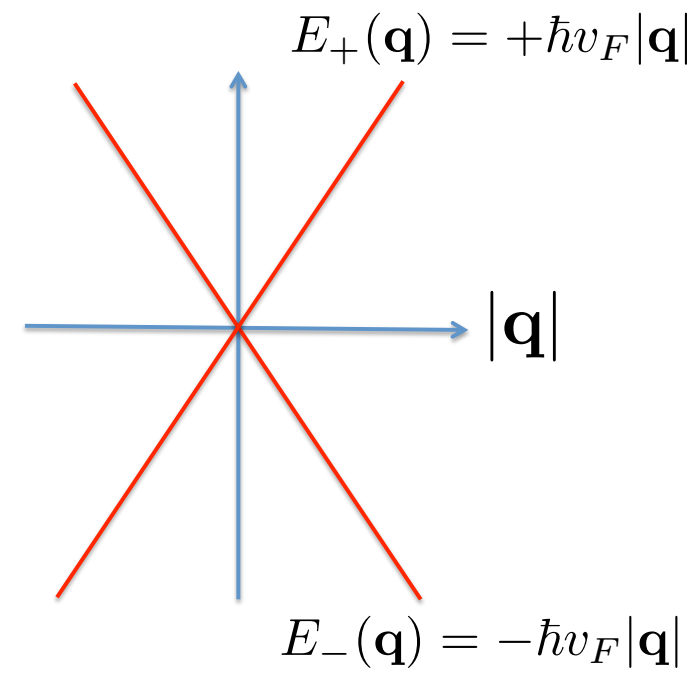

(a)

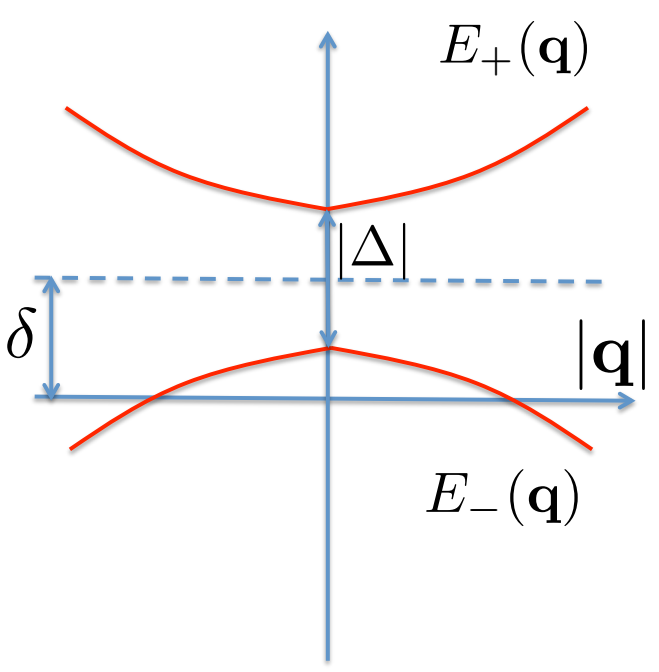

(b)

Figure S5: (a) The spectrum of the free Hamiltonian Eq.(S10) near one of the Dirac points. (b) The spectrum of the Hamiltonian Eq.(S27) with on-site interaction, after Eq.(S29), near one of the Dirac points. 
with $V_{A}$ and $V_{B}$ defined by Eq.(S20).

By applying the same technique as explained in the previous section for the free Hamiltonian, we obtain the representation of the interaction term Eq.(S13) in crystal momentum space in the vicinity of both Dirac points $\mathbf{k}=\mathbf{K}_{D}^{( \pm)}+\mathbf{q}$

$$
\hat{H}_{i n t}=\sum_{\sigma, \mathbf{q}} \hat{\Psi}_{\sigma}^{\dagger}(\mathbf{q})\left(\begin{array}{cccc}
V_{A} & 0 & 0 & 0 \\
0 & V_{B} & 0 & 0 \\
0 & 0 & V_{B} & 0 \\
0 & 0 & 0 & V_{A}
\end{array}\right) \hat{\Psi}_{\sigma}(\mathbf{q}),
$$

with the constants $V_{A}$ and $V_{B}$ defined in Eq.(S20). Let us now define the parameters:

$$
\Delta=\left(V_{A}-V_{B}\right), \quad \delta=\frac{1}{2}\left(V_{A}+V_{B}\right) .
$$

Substituting these definitions into Eq.(S22), the interaction Hamiltonian can be recast into the combination:

$$
\hat{H}_{\text {int }}=\hat{H}_{\text {mass }}+\hat{H}_{V}
$$

with

$$
\begin{aligned}
\hat{H}_{\text {mass }} & =\sum_{\sigma, \mathbf{q}} \hat{\Psi}_{\sigma}^{\dagger}(\mathbf{q})\left(\begin{array}{cccc}
\Delta / 2 & 0 & 0 & 0 \\
0 & -\Delta / 2 & 0 & 0 \\
0 & 0 & -\Delta / 2 & 0 \\
0 & 0 & 0 & \Delta / 2
\end{array}\right) \hat{\Psi}_{\sigma}(\mathbf{q}) \\
& =\sum_{\sigma, \mathbf{q}} \hat{\Psi}_{\sigma}^{\dagger}(\mathbf{q})\left(\begin{array}{cc}
\frac{\Delta}{2} \hat{\sigma}_{z} & 0 \\
0 & -\frac{\Delta}{2} \hat{\sigma}_{z}
\end{array}\right) \hat{\Psi}_{\sigma}(\mathbf{q})
\end{aligned}
$$

and

$$
\begin{aligned}
\hat{H}_{V} & =\sum_{\sigma, \mathbf{q}} \hat{\Psi}_{\sigma}^{\dagger}(\mathbf{q})\left(\begin{array}{cccc}
\delta & 0 & 0 & 0 \\
0 & \delta & 0 & 0 \\
0 & 0 & \delta & 0 \\
0 & 0 & 0 & \delta
\end{array}\right) \hat{\Psi}_{\sigma}(\mathbf{q}) \\
& =\sum_{\sigma, \mathbf{q}} \hat{\Psi}_{\sigma}^{\dagger}(\mathbf{q})\left(\begin{array}{cc}
\delta \hat{\sigma}_{0} & 0 \\
0 & \delta \hat{\sigma}_{0}
\end{array}\right) \hat{\Psi}_{\sigma}(\mathbf{q}),
\end{aligned}
$$

where $\left\{\hat{\sigma}_{j}\right\}$ are the Pauli matrices defined in Eq.(S11). Combining the free Hamiltonian Eq.(S10) with the interaction terms in Eq.(S25) and Eq.(S26), we have the total Hamiltonian that includes the contribution from both Dirac points,

$$
\begin{aligned}
\hat{H} & =\hat{H}_{0}+\hat{H}_{i n t} \\
& =\sum_{\mathbf{q}, \sigma} \hat{\Psi}_{\sigma}^{\dagger}(\mathbf{q})\left[\hat{\tau}_{z} \otimes\left(\hbar v_{F} \vec{\sigma} \cdot \mathbf{q}+\frac{\Delta}{2} \hat{\sigma}_{z}\right)+\delta \hat{\tau}_{0} \otimes \hat{\sigma}_{0}\right] \hat{\Psi}_{\sigma}(\mathbf{q}) .
\end{aligned}
$$


Here, we have defined a second set of Pauli matrices that account for the two valleys $\mathbf{K}_{D}^{ \pm}$

$$
\hat{\tau}_{z}=\left(\begin{array}{cc}
1 & 0 \\
0 & -1
\end{array}\right), \quad \hat{\tau}_{0}=\left(\begin{array}{ll}
1 & 0 \\
0 & 1
\end{array}\right) .
$$

Exact diagonalization of the Hamiltonian in Eq.(S27) leads to the following energy eigenvalues:

$$
E_{ \pm}(\mathbf{q})=\delta \pm \hbar v_{F} \sqrt{\mathbf{q}^{2}+\left(\frac{\Delta}{2 \hbar v_{F}}\right)^{2}}
$$

Notice that Eq.(S29) predicts a shift $\delta$ of the Dirac cone, as well as a gap opening $|\Delta|$ (notice the absolute value), with these parameters defined after Eq.(S23), in terms of the constants defined in Eq.(S20). A graphical representation of Eq.(S29) is depicted in Fig.S5.

To illustrate how this mechanism works, let us consider a material monolayer with two different types of atoms (1 and 2). The electrostatic environment for each carbon atom in the unit cell of graphene will thus depend on its relative position with respect to the other material, either on top of a type 1 or type 2 atom, or on top of a vacancy site. This situation is captured by the effective potential acting over an $A$ or $B$-carbon atom of the graphene sub-lattices, that we define as

$$
V\left(\mathbf{r}_{(A, B)}^{(j)}\right)=\left\{\begin{array}{cc}
U_{1}, & \text { if } \mathbf{r}_{(A, B)}^{(j)} \text { over atom } 1 \\
U_{2}, & \text { if } \mathbf{r}_{(A, B)}^{(j)} \text { over atom } 2 \\
0, & \text { if } \mathbf{r}_{(A, B)}^{(j)} \text { over vacancy }
\end{array}\right.
$$

Let us now consider a heterostructure with $n=n_{A}=n_{B}$ graphene carbon atoms in the enlarged unit cell, such that $n_{A}^{(1)}, n_{B}^{(1)} A$ and $B$-atoms are on top of a type- 1 atom of the substrate, respectively, while $n_{A}^{(2)}, n_{B}^{(2)}$ are on top of a type-2 atom of the substrate. Therefore, the number of carbon atoms of $A$ and $B$-graphene sub lattices that go on top of vacancy sites in the substrate will be $n_{A}^{(0)}=n-n_{A}^{(1)}-n_{A}^{(2)}$ and $n_{B}^{(0)}=n-n_{B}^{(1)}-n_{B}^{(2)}$. We evaluate the coefficients defined in Eq.(S20)

$$
\begin{aligned}
V_{A} & =\frac{1}{n}\left(n_{A}^{(0)} \cdot 0+n_{A}^{(1)} U_{1}+n_{A}^{(2)} U_{2}\right), \\
V_{B} & =\frac{1}{n}\left(n_{B}^{(0)} \cdot 0+n_{B}^{(1)} U_{1}+n_{B}^{(2)} U_{2}\right) .
\end{aligned}
$$

Using the values obtained in Eq.(S31), we calculate the parameters $\Delta$ and $\delta$ defined in Eq.(S23),

$$
\begin{aligned}
\Delta & =\left(V_{A}-V_{B}\right) \\
& =\frac{1}{n}\left[\left(n_{A}^{(1)}-n_{B}^{(1)}\right) U_{1}+\left(n_{A}^{(2)}-n_{B}^{(2)}\right) U_{2}\right] \\
\delta & =\frac{1}{2}\left(V_{A}+V_{B}\right) \\
& =\frac{1}{2 n}\left[\left(n_{A}^{(1)}+n_{B}^{(1)}\right) U_{1}+\left(n_{A}^{(2)}+n_{B}^{(2)}\right) U_{2}\right] .
\end{aligned}
$$


It follows as an immediate conclusion from Eq.(S32) that a necessary condition for Dirac cone splitting in graphene is symmetry breaking between the graphene sublattices $A, B$, provided that at least one of the numbers, $n_{A}^{(1)}-n_{B}^{(1)} \neq 0$ or $n_{A}^{(2)}-n_{B}^{(2)} \neq 0$. Let us now apply this simple stoichiometric analysis to interpret some of the cases presented in the main text of the article. A more extensive analysis is summarized in Table S1.

\section{Case 1: $g r / h-B N$ with $A A$ stacking (Fig. 2(a))}

From Fig. 2(a), clearly the unit cell has $n=n_{A}=n_{B}=1$ carbon atoms from each graphene sublattice, while the $h-B N$ has two different types of atoms, i.e. boron (1) and nitrogen (2). No carbon atoms are lying on the top of a vacancy in this stacking. Therefore, $n_{A}^{(0)}=n_{B}^{(0)}=0$, while $n_{A}^{(1)}=1, n_{B}^{(1)}=0$, $n_{A}^{(2)}=0$, and $n_{B}^{(2)}=1$. Substituting into Eq.(S32), we obtain

$$
|\Delta|=\frac{1}{1}\left|(1-0) U_{1}+(0-1) U_{2}\right|=\left|U_{1}-U_{2}\right|
$$

which predicts a Dirac cone splitting with a finite gap, in agreement with the numerical calculation.

\section{Case 2: $g r / h-B N$ with $A B$ stacking (Fig. 2(b))}

From Fig. 2(b), clearly the unit cell has $n=n_{A}=n_{B}=1$ carbon atoms from each graphene sublattice, while the $h-B N$ has two different types of atoms, i.e. boron (1) and nitrogen (2). In this stacking configuration, one fourth of a $B$-carbon atom is lying at each corner of the unit cell, on top of a vacancy site, and hence $n_{B}^{(0)}=4 \cdot(1 / 4)=1$, while $n_{A}^{(0)}=0$. The single $A$-carbon atom is lying on top of a boron atom, hence $n_{A}^{(2)}=1$, while $n_{B}^{(2)}=0$. Finally, no carbon atom is on top of the boron atom, hence $n_{A}^{(1)}=n_{B}^{(1)}=0$. Substituting into Eq.(S32), we obtain

$$
|\Delta|=\frac{1}{1}\left|(0-0) U_{1}+(1-0) U_{2}\right|=\left|U_{2}\right|,
$$

which predicts a Dirac cone splitting, with a finite energy gap, as verified form ab-initio calculations.

\section{Case 3: $g r / h-C_{3} N_{4}$ with $A A$ stacking (Fig. 2(c))}

From Fig. 2(c), clearly the unit cell has $n=n_{A}=n_{B}=9$ carbon atoms from each graphene sublattice, while the $h-C_{3} N_{4}$ has two different types of atoms, carbon (1) and nitrogen (2). Clearly $n_{A}^{(1)}=6$ A-atoms are on top of carbon, while $n_{B}^{(1)}=0 \mathrm{~B}$-atoms are on top of carbon. On the other hand, no A-atom is on top of nitrogen $n_{A}^{(2)}=0$, while $n_{B}^{(2)}=8 \mathrm{~B}$ atoms are on top of nitrogen. Finally, $n_{A}^{(0)}=3 A$-atoms and $n_{B}^{(0)}=1$ $B$-atom are on top of vacancy sites. Therefore, from Eq.(S32) we have

$$
|\Delta|=\frac{1}{9}\left|(6-0) U_{1}+(0-8) U_{2}\right|=\frac{\left|6 U_{1}-8 U_{2}\right|}{9},
$$

which predicts a Dirac cone splitting, with a finite energy gap, as verified from ab-initio calculations. 


\section{Case 4: $g r / h-C_{3} N_{4}$ with $A B$ stacking (Fig. 2(d))}

From Fig. 2(d), clearly the unit cell has $n=n_{A}=n_{B}=9$ carbon atoms from each graphene sublattice, while the $h-C_{3} N_{4}$ has two different types of atoms, carbon (1) and nitrogen (2). There are no $A$-atoms on top of carbon, $n_{A}^{(1)}=0$, while $n_{B}^{(1)}=6 \mathrm{~B}$-atoms are on top of carbon. On the other hand, no $A$ nor $B$-atoms are on top of nitrogen, $n_{A}^{(2)}=0, n_{B}^{(2)}=0$. Finally, $n_{A}^{(0)}=9 A$-atoms and $n_{B}^{(0)}=3 B$-atoms are on top of vacancy sites. Therefore, from Eq.(S32) we have

$$
|\Delta|=\frac{1}{9}\left|(0-6) U_{1}+(0-0) U_{2}\right|=\frac{2\left|U_{1}\right|}{3},
$$

which predicts a Dirac cone splitting, with a finite energy gap, as verified from $a b$-initio calculations.

\section{Case 5: $g r / C_{3} N$ with $A A$ stacking (Fig. 2(e))}

From Fig. 2(e), clearly the unit cell has $n=n_{A}=n_{B}=4$ carbon atoms from graphene, while the substrate has two different types of atoms, carbon (1) and nitrogen (2). Clearly $n_{A}^{(1)}=3$ A-atoms are on top of carbon, while $n_{B}^{(1)}=4 \cdot(1 / 4)+2 \cdot(1 / 2)=3 \mathrm{~B}$-atoms are on top of carbon. On the other hand, a single A-atom is on top of nitrogen $n_{A}^{(2)}=1$, while $n_{B}^{(2)}=2 \cdot(1 / 2)=1 \mathrm{~B}$ atoms are on top of nitrogen. Neither A nor B atoms are on top of vacancies. Hence, form Eq.(S32) we have

$$
|\Delta|=\frac{1}{4}\left|(3-3) U_{1}+(1-1) U_{2}\right|=0,
$$

and hence, no Dirac cone splitting is predicted for this case, in agreement with the ab-initio calculations.

\section{Case 6: $g r / C_{3} N$ with $A B$ stacking (Fig. 2(f))}

From Fig. 2(f), clearly the unit cell has $n=n_{A}=n_{B}=4$ carbon atoms from graphene, while the substrate has two different types of atoms, carbon (1) and nitrogen (2). Clearly $n_{A}^{(1)}=3$ A-atoms are on top of carbon, while no B-atoms are on top of carbon $n_{B}^{(1)}=0$. On the other hand, a single A-atom is on top of nitrogen $n_{A}^{(2)}=1$, while no B-atoms are on top of nitrogen $n_{B}^{(2)}=0$. Finally, no A toms are on top of vacancies $n_{A}^{(0)}=0$, but $n_{B}^{(0)}=4 \cdot(1 / 4)+4 \cdot(1 / 2)=3 \mathrm{~B}$-atoms are on top of vacancies. Hence, from Eq.(S32) we have

$$
|\Delta|=\frac{1}{4}\left|(3-0) U_{1}+(1-0) U_{2}\right|=\frac{\left|3 U_{1}+U_{2}\right|}{4},
$$

and hence, a Dirac cone splitting with a finite gap is predicted, in agreement with the ab-initio calculations. 
(a)

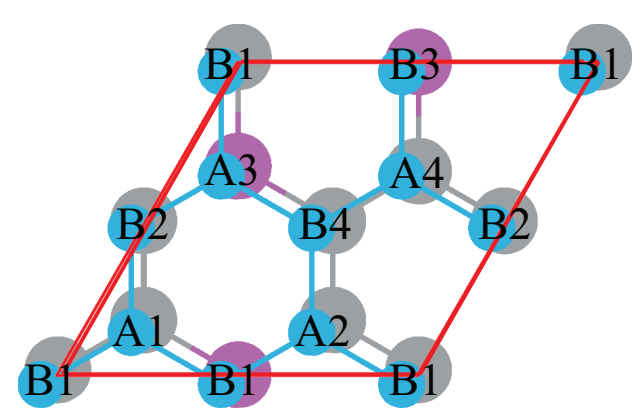

(c)

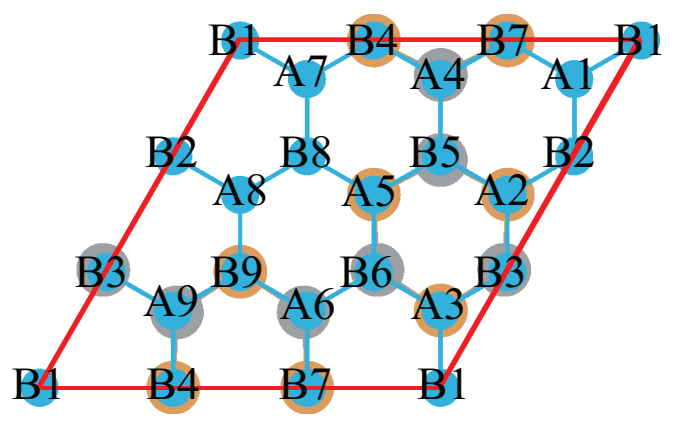

(e)

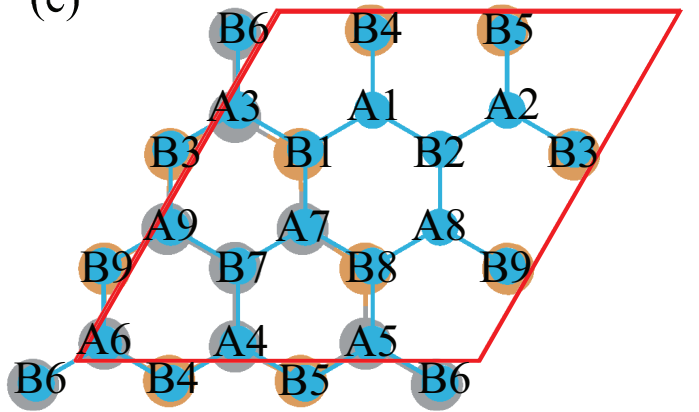

(b)

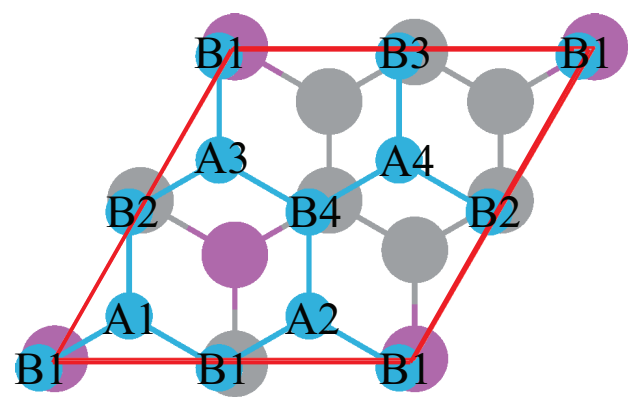

(d)

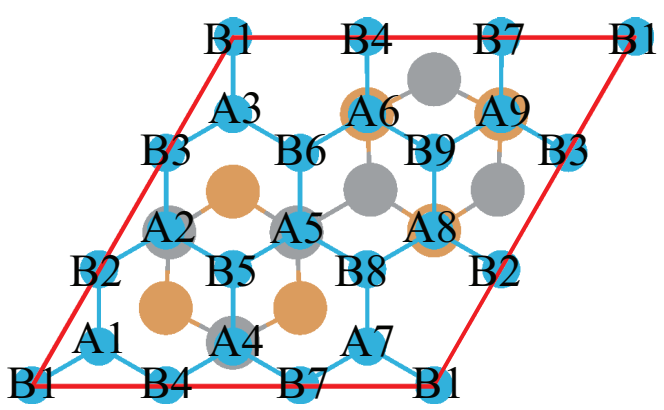

(f)

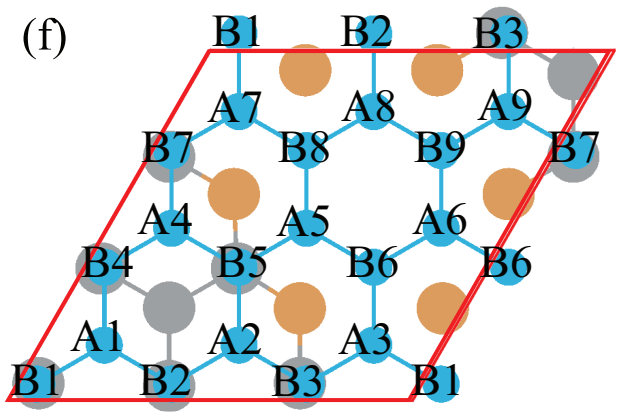

Figure S6: Top view of (a) AA and (b) $\mathrm{AB}$ stacking of $\mathrm{gr} / \mathrm{BC}_{3}$, (c) AA and (d) $\mathrm{AB}$ stacking of gr/ $\mathrm{C}_{3} \mathrm{~N}_{3}$, (e) AA and (d) $\mathrm{AB}$ stacking of gr/h- $\mathrm{C}_{4} \mathrm{~N}_{3}$ heterostructures. Yellow, brown, green, and blue filled circles represent $\mathrm{C}$ (graphene), $\mathrm{C}$ (substrate layer), $\mathrm{B}$, and $\mathrm{N}$ atoms, respectively. The size of circles in the bottom layers have been made larger to show the kind of atoms that are present below $\mathrm{C}$ atoms in the graphene layer. 
(a)

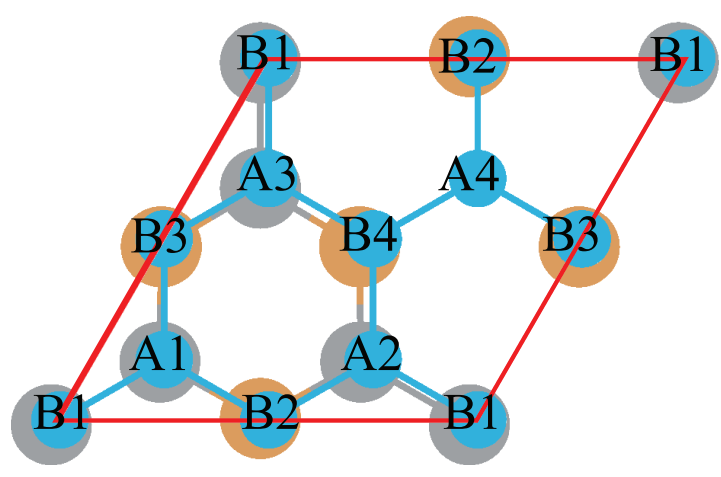

(c)

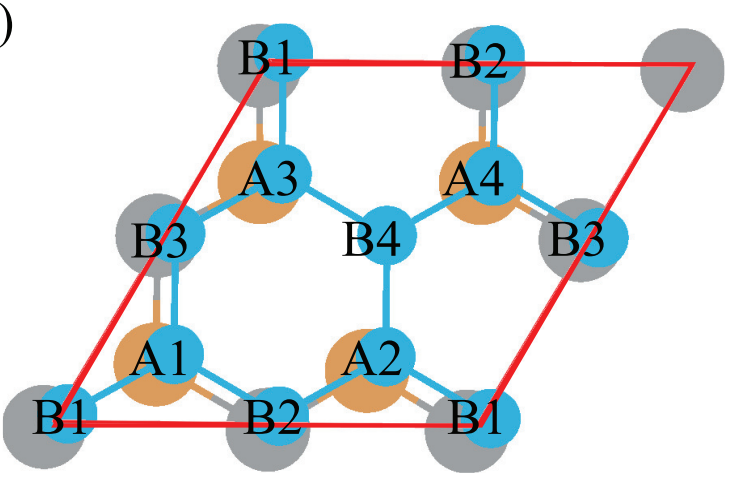

(e)

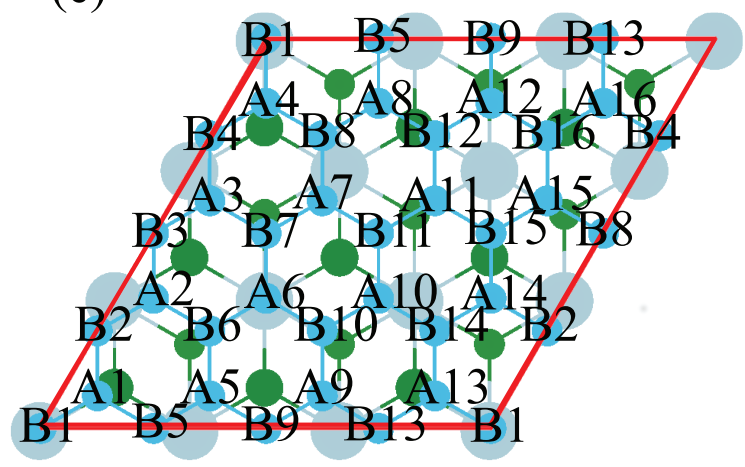

(b)

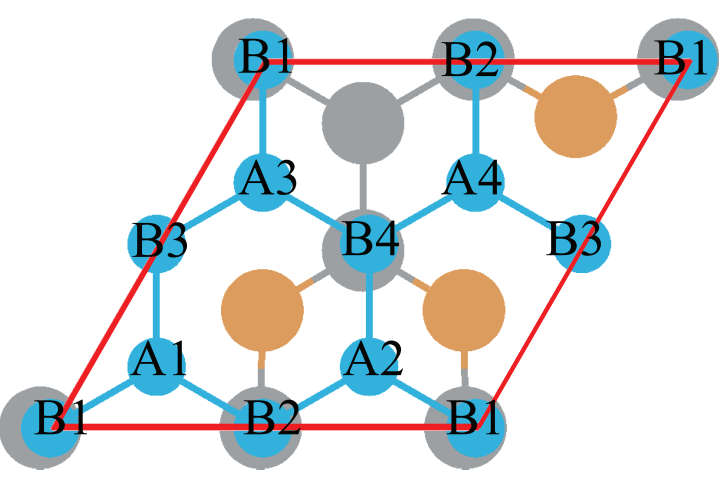

(d)

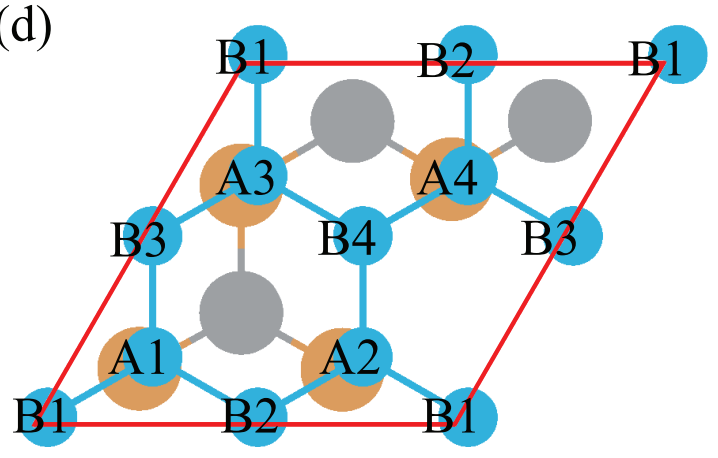

(f)

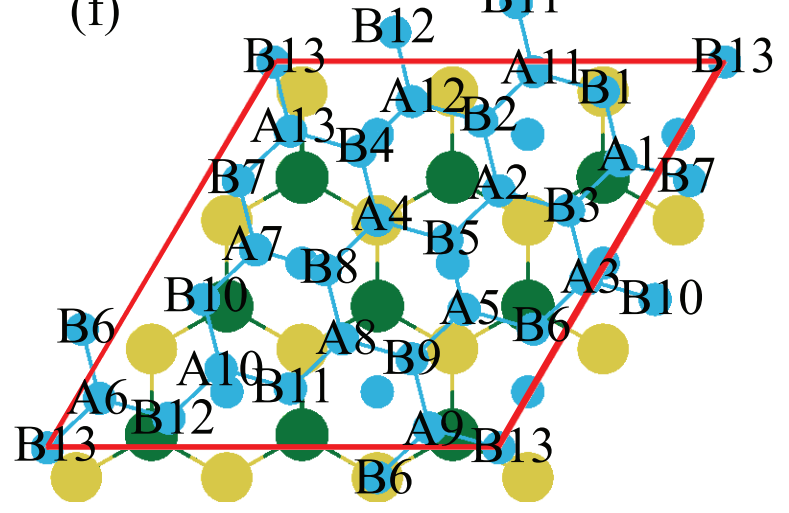

Figure S7: Top view of (a) AA and (b) AB stacking of gr/t- $\mathrm{C}_{4} \mathrm{~N}_{3}$, (c) AA and (d) AB stacking of gr/t- $\mathrm{C}_{3} \mathrm{~N}_{4}$, (e) $\mathrm{gr} / \mathrm{HfO}_{2}$, and (d) $\mathrm{gr} / \mathrm{Ti}_{2} \mathrm{CO}_{2}$ heterostructures. Yellow, brown, blue, cyan, dark-grey, and dark-green filled circles represent $\mathrm{C}$ (graphene), $\mathrm{C}$ (substrate layers), $\mathrm{N}, \mathrm{Hf}$, Ti, and $\mathrm{O}$ atoms, respectively. The size of circles in the bottom layers have been made larger to show the kind of atoms that are present below $\mathrm{C}$ atoms in the graphene layer. 
(a)

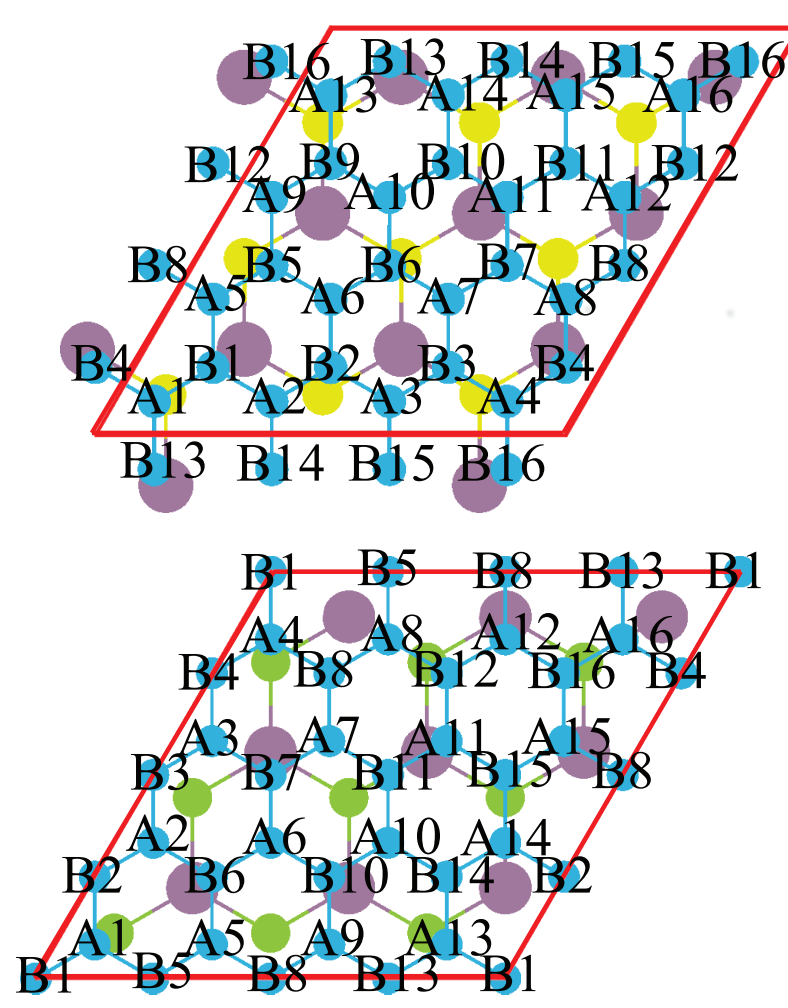

(e)

(c)

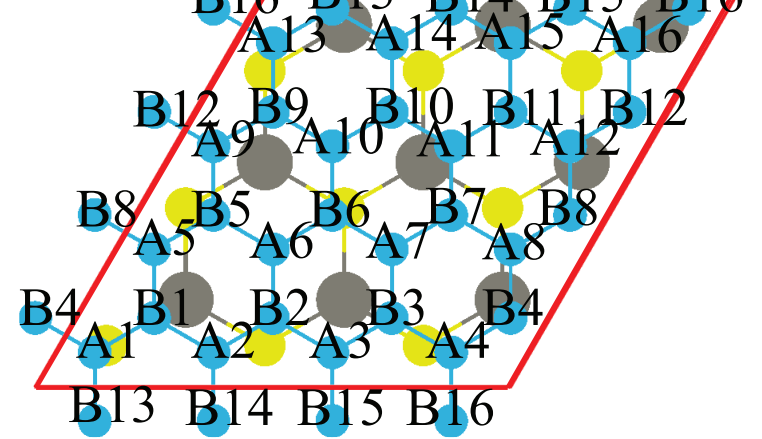

(b)

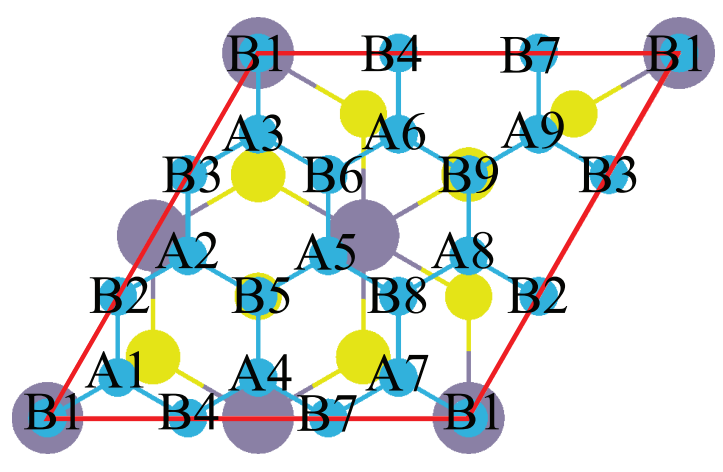

(d)

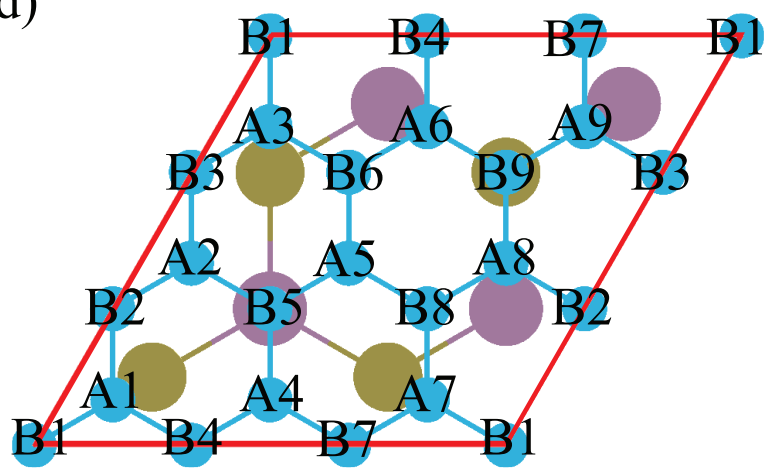

(f)

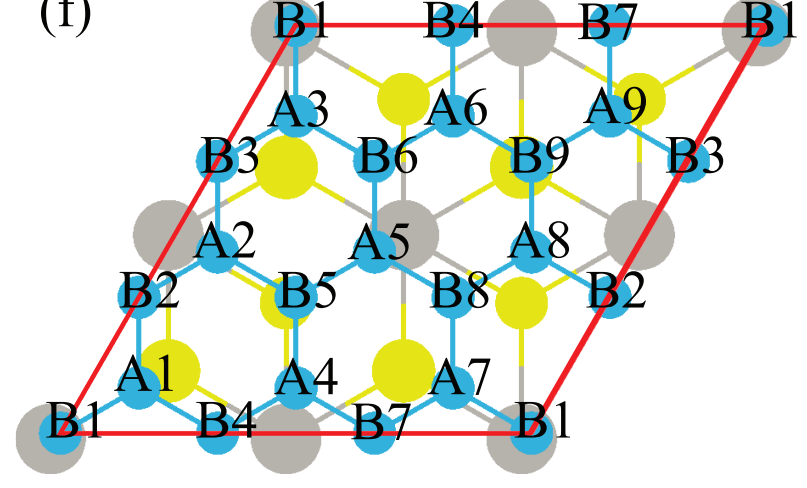

Figure S8: Top view of (a) gr/MoS ${ }_{2}$, (b) gr/SnS ${ }_{2}$, (c) gr/ $\mathrm{MoSe}_{2}$, (d) gr/MoTe 2 , (e) gr/WS $\mathrm{Wh}_{2}$, and (f) gr/ $/ \mathrm{PtS}_{2}$ heterostructures. Yellow, voilet, indigo, grey, orange, lime-green, and umber filled circles represent $\mathrm{C}$, Mo, $\mathrm{Sn}, \mathrm{Pt}, \mathrm{S}, \mathrm{Se}$, and Te atoms, respectively. The size of circles in the bottom layers have been made larger to show the kind of atoms that are present below $\mathrm{C}$ atoms in the graphene layer. 
(a)

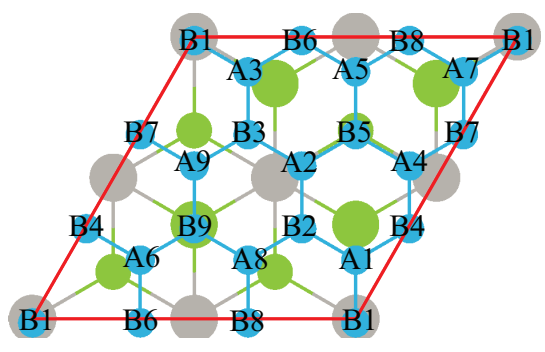

(b)

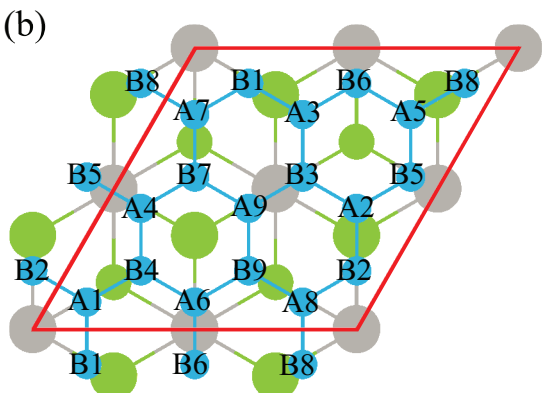

(c)

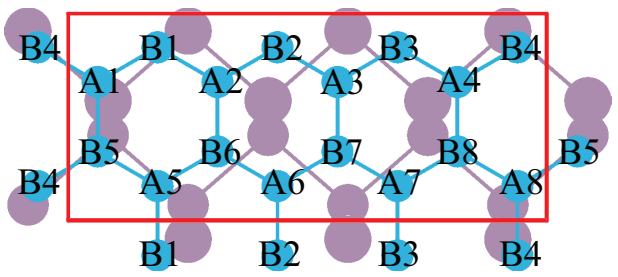

Figure S9: Top view of (a) stacking 1 and (b) stacking 2 of gr/PtSe ${ }_{2}$, and (c) gr/phos heterostructures. Yellow, grey, lime-green, and pink filled circles represent $\mathrm{C}, \mathrm{Pt}, \mathrm{Se}$, and $\mathrm{P}$, atoms respectively. The size of circles in the bottom layers have been made larger to show the kind of atoms that are present below $\mathrm{C}$ atoms in the graphene layer.
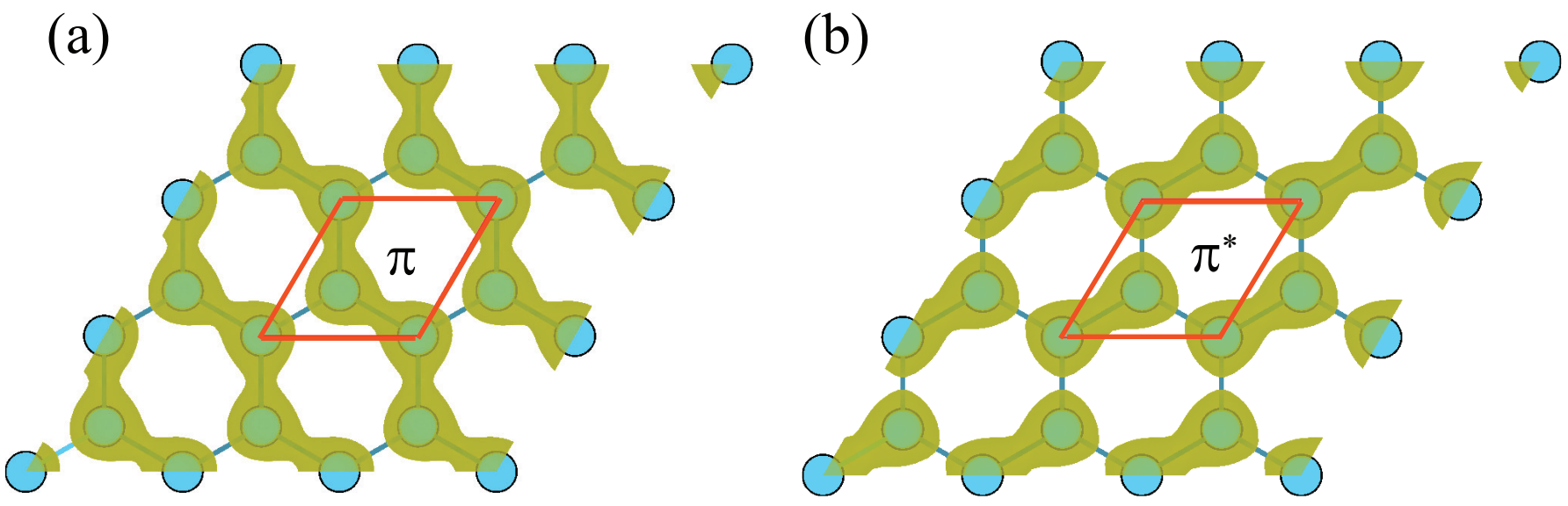

Figure S10: Band-decomposed charge density of (a) valence band maxima (VBM), which is a $\pi$ state, and (b) conduction band minima $(\mathrm{CBM})$ of graphene, which is a $\pi^{*}$ state. 

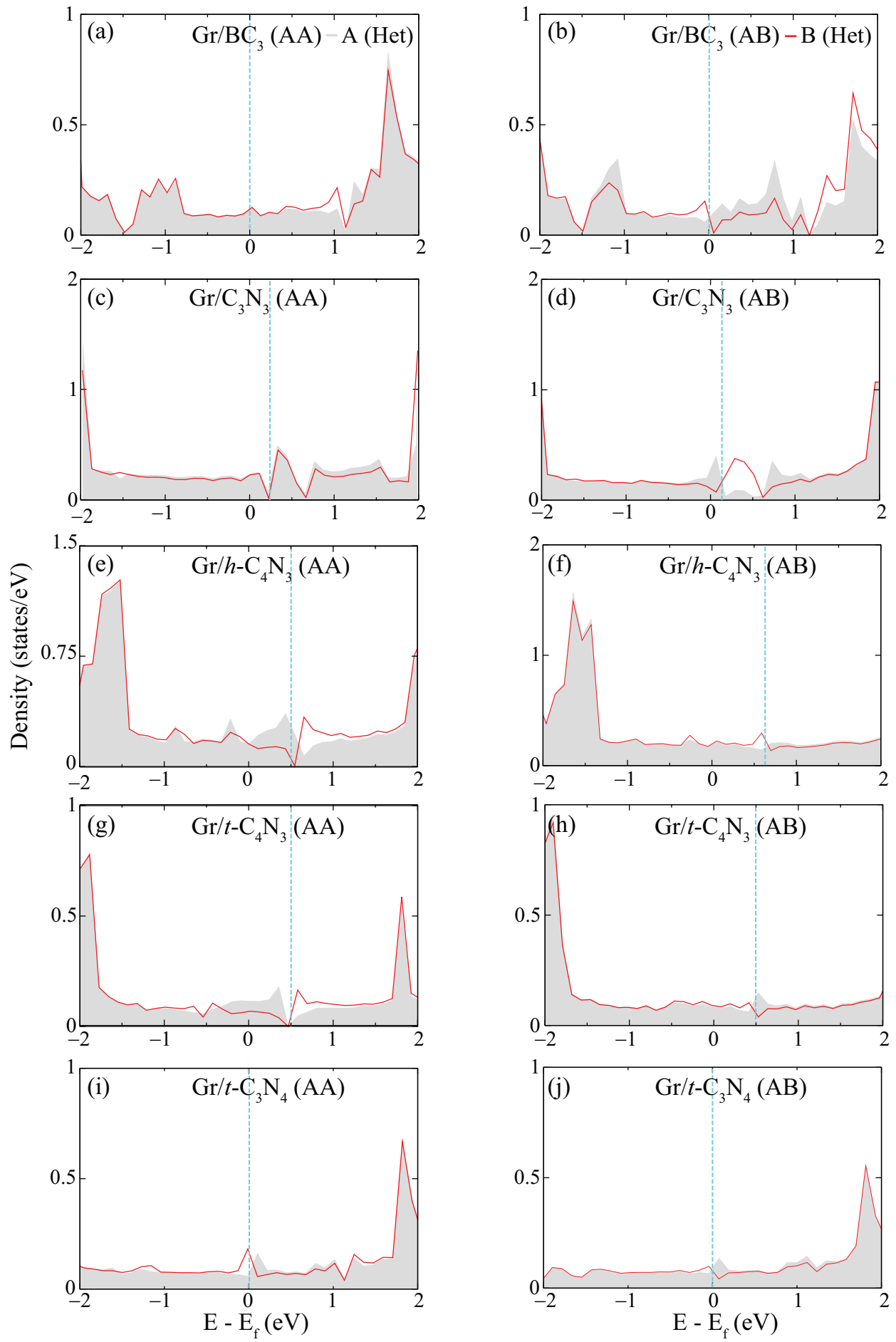

Figure S11: LDOS projected onto combined- $A$ (denoted by grey-filled region) and $B$ (denoted by solid red curve) sublattices of graphene present on (a) $\mathrm{BC}_{3}$ (AA stacking), (b) $\mathrm{BC}_{3}$ (AB stacking), (c) $\mathrm{C}_{3} \mathrm{~N}_{3}$ ( $\mathrm{AA}$ stacking), (d) $\mathrm{C}_{3} \mathrm{~N}_{3}$ (AB stacking), (e) $h-\mathrm{C}_{4} \mathrm{~N}_{3}$ (AA stacking), (f) $h-\mathrm{C}_{4} \mathrm{~N}_{3}$ (AB stacking), (g) $t-\mathrm{C}_{4} \mathrm{~N}_{3}(\mathrm{AA}$ stacking), (h) $t-\mathrm{C}_{4} \mathrm{~N}_{3}$ (AB stacking), (i) $t-\mathrm{C}_{3} \mathrm{~N}_{4}$ (AA stacking), (j) $t-\mathrm{C}_{3} \mathrm{~N}_{4}$ (AB stacking) substrates. The cyan-dashed line indicate the position of Dirac cone of graphene $\left(\mathrm{E}_{D}\right)$. 

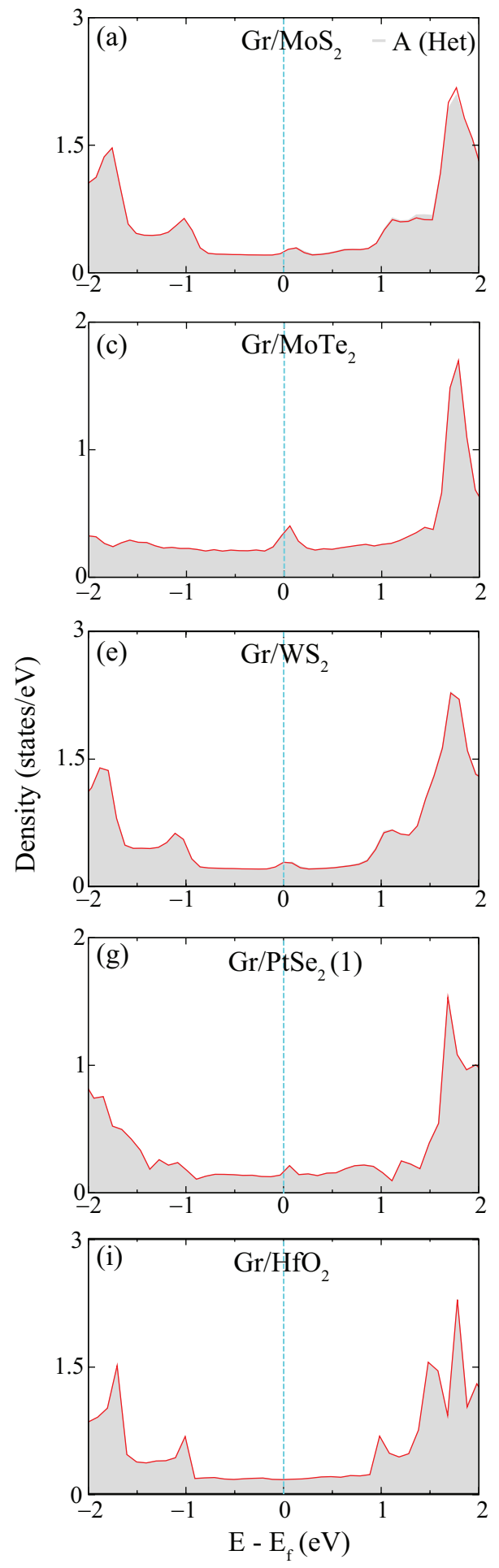
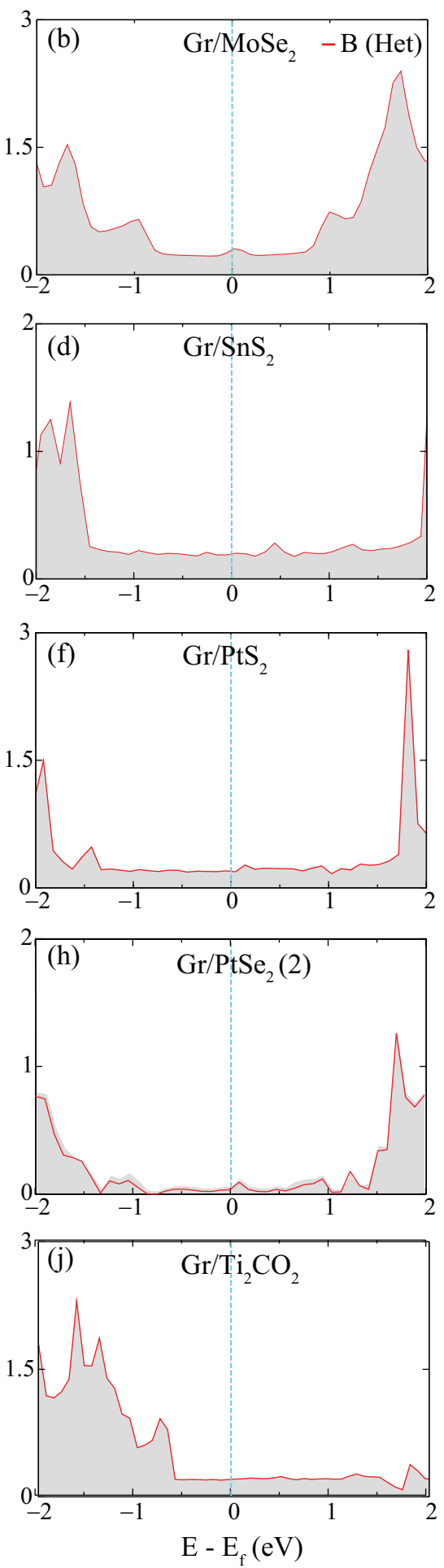

Figure S12: LDOS projected onto combined- $A$ (denoted by grey-filled region) and $B$ (denoted by solid red curve) sublattices of graphene present on (a) $\mathrm{MoS}_{2}$, (b) $\mathrm{MoSe}_{2}$, (c) $\mathrm{MoTe}_{2}$, (d) $\mathrm{SnS}_{2}$, (e) $\mathrm{WS}_{2}$, (f) $\mathrm{PtS}_{2}$, (g) $\mathrm{PtSe}_{2}$ (stacking 1), (h) $\mathrm{PtSe}_{2}$ (stacking 2), (i) $\mathrm{HfO}_{2}$, (j) $\mathrm{Ti}_{2} \mathrm{CO}_{2}$ substrates. The cyan-dashed line indicate the position of Dirac cone of graphene $\left(\mathrm{E}_{D}\right)$. 

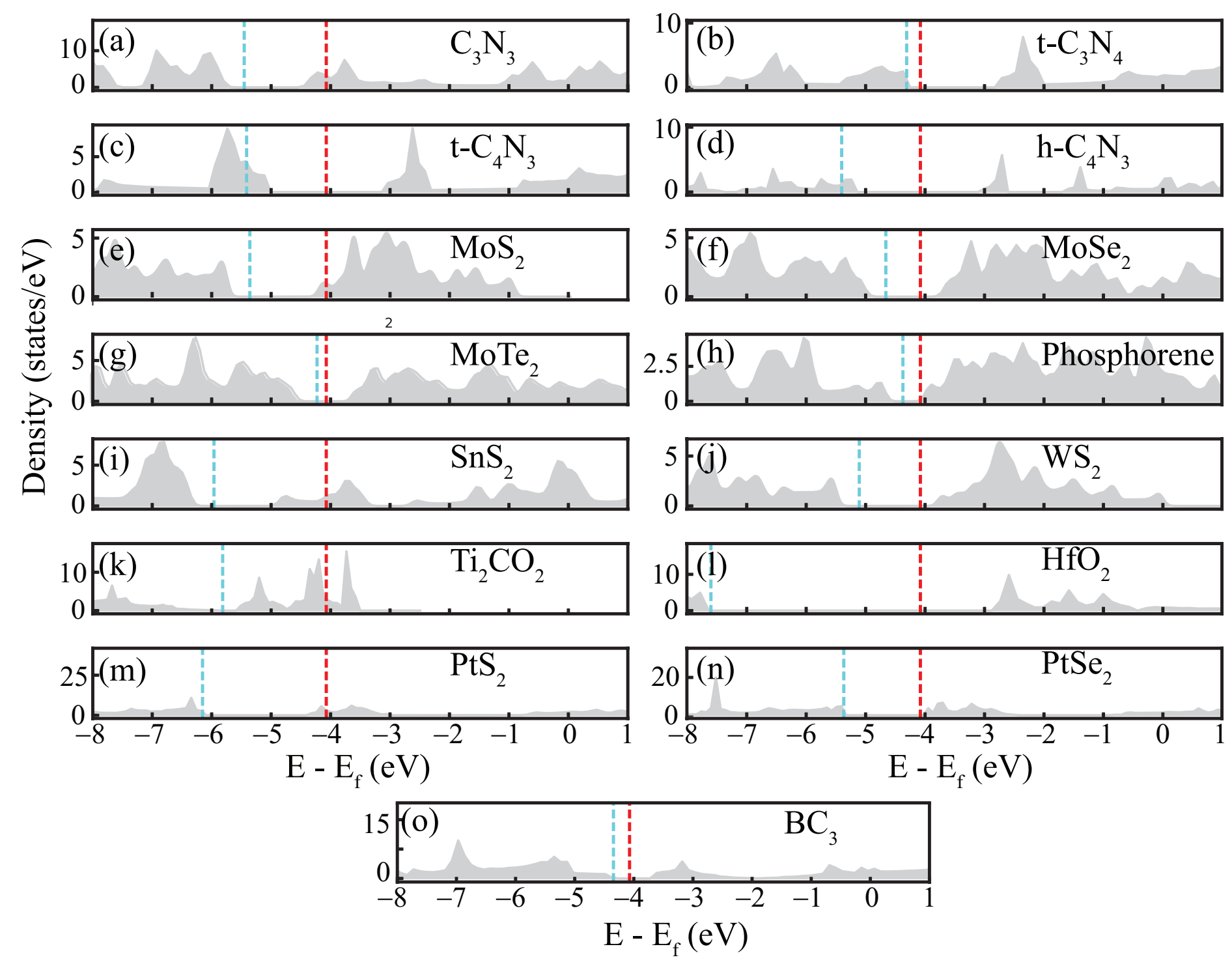

Figure S13: Density of states (DOS) of isolated (a) $\mathrm{C}_{3} \mathrm{~N}_{3}$, (b) $t-\mathrm{C}_{3} \mathrm{~N}_{4}$, (c) $h-\mathrm{C}_{4} \mathrm{~N}_{3}$, (d) $t-\mathrm{C}_{4} \mathrm{~N}_{3}$, (e) $\mathrm{MoS}_{2}$, (f) $\mathrm{MoSe}_{2}$, (g) $\mathrm{MoTe}_{2}$, (h) phosphorene, (i) $\mathrm{SnS}_{2}$, (j) $\mathrm{WS}_{2}$, (k) $\mathrm{Ti}_{2} \mathrm{CO}_{2}$, (l) $\mathrm{HfO}_{2}$, (m) $\mathrm{PtS}_{2}$, (n) $\mathrm{PtSe}_{2}$, and (o) $\mathrm{BC}_{3}$ monolayers, with respect to the respective vacuum levels. The cyan and red-dashed vertical lines denote the fermi levels of corresponding layers and fermi level of isolated graphene $\left(\mathrm{E}_{D}\right)$, respectively. $\mathrm{E}_{D}$ is also aligned with respect to its vacuum level. 
(a)

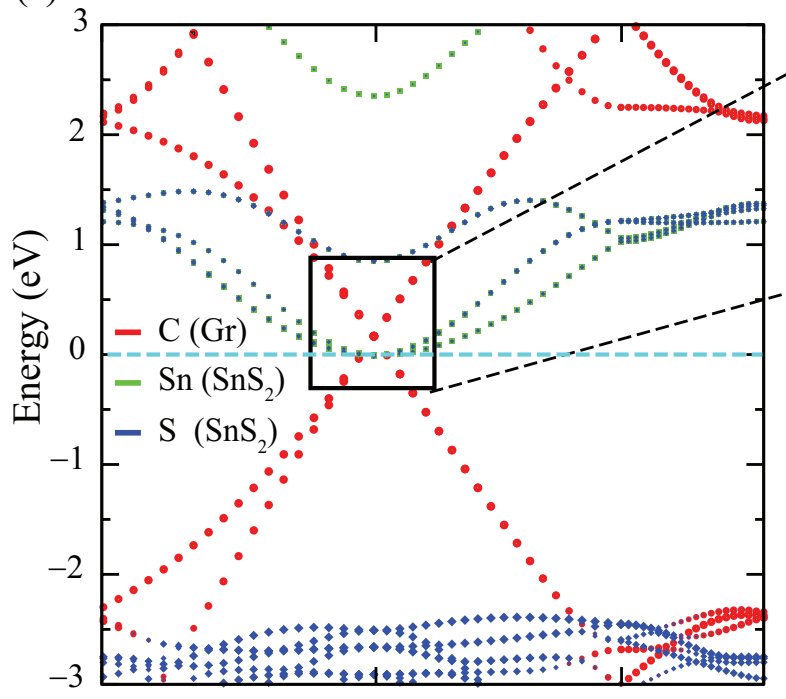

(b)

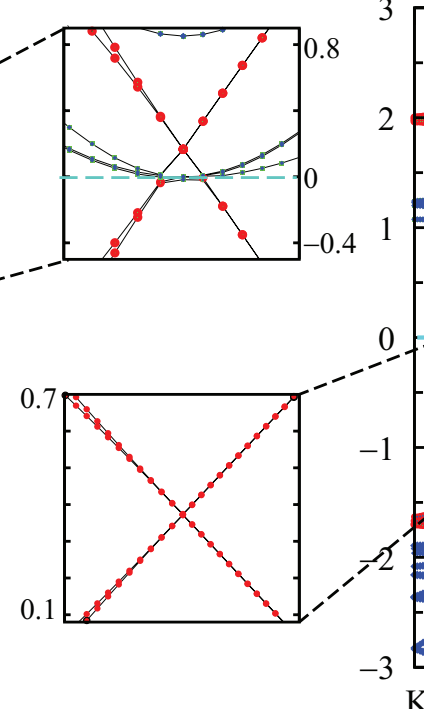

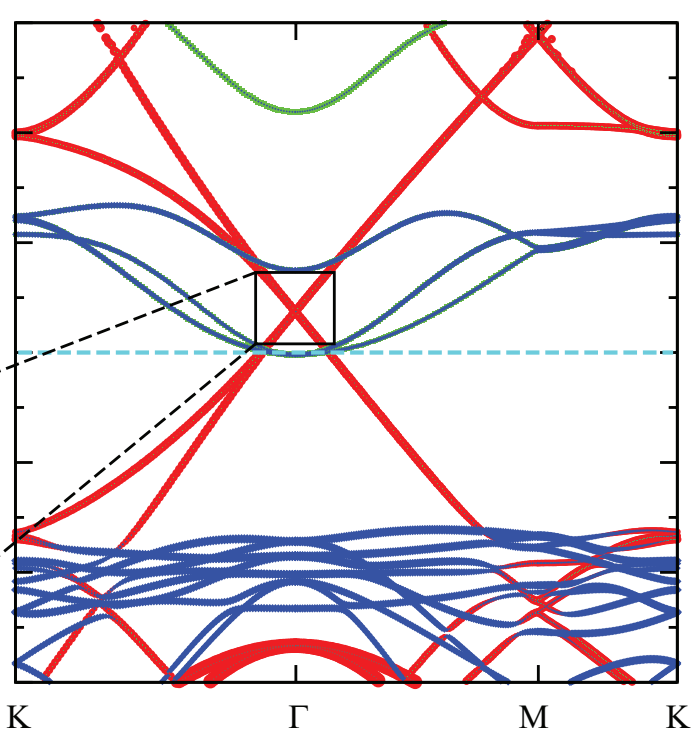

Figure S14: Electronic band structures of gr/SnS 2 heterostructure under (a) HSE06 and (b) PBE calculations.

(a)

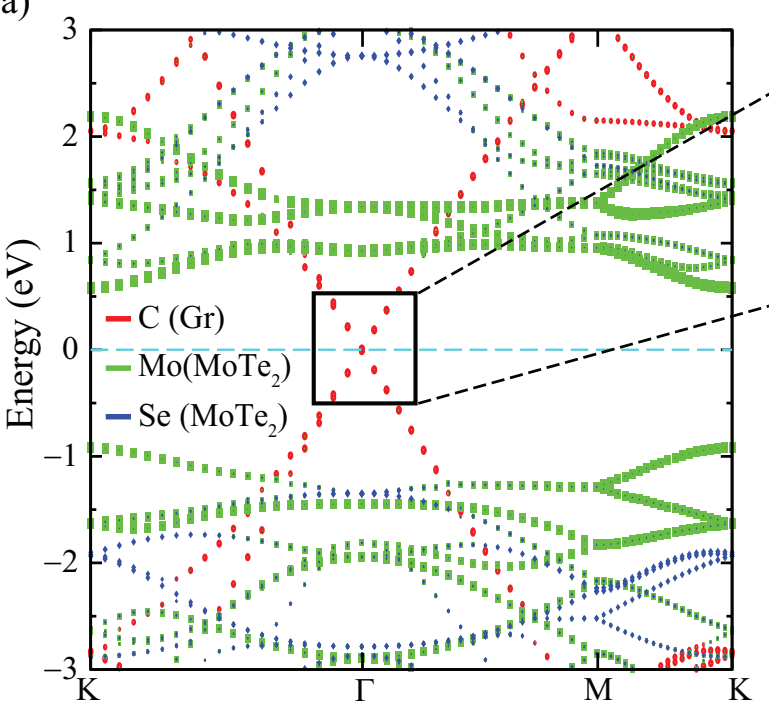

(b)

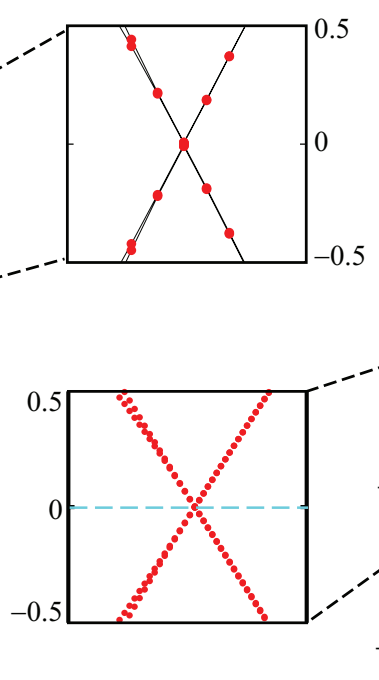

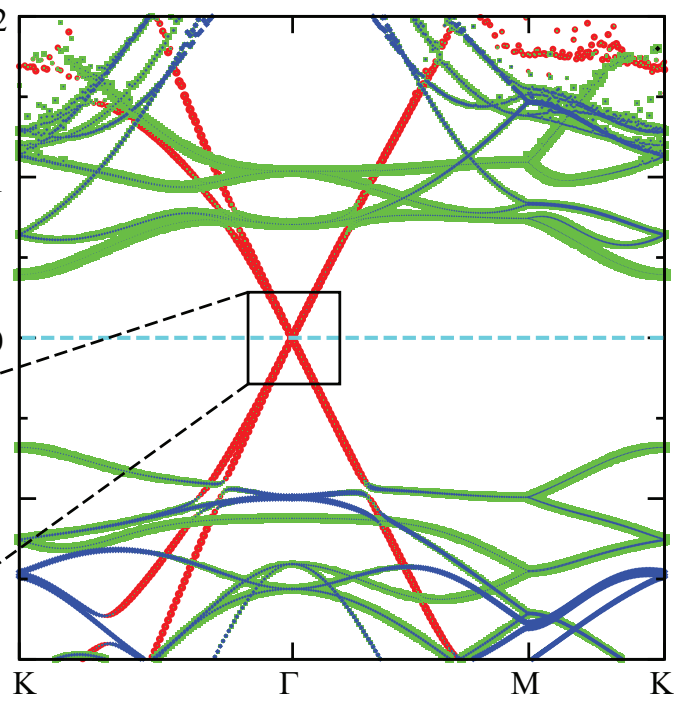

Figure S15: Electronic band structures of gr/MoTe ${ }_{2}$ heterostructure under (a) HSE06 and (b) PBE calculations. 

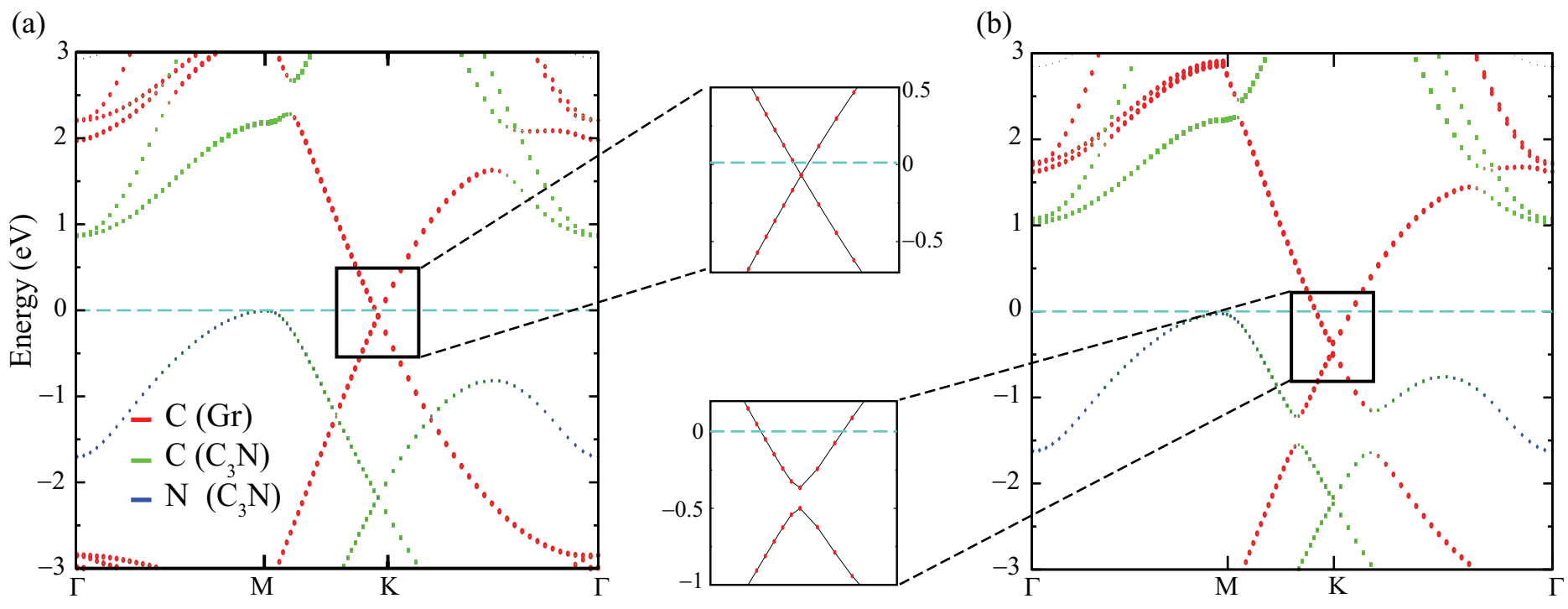

Figure S16: Electronic band structures of (a) $\mathrm{AA}$ and (b) $\mathrm{AB}$ stacking of $\mathrm{gr} / \mathrm{C}_{3} \mathrm{~N}$ heterostructures under HSE06 calculations. 
(a)
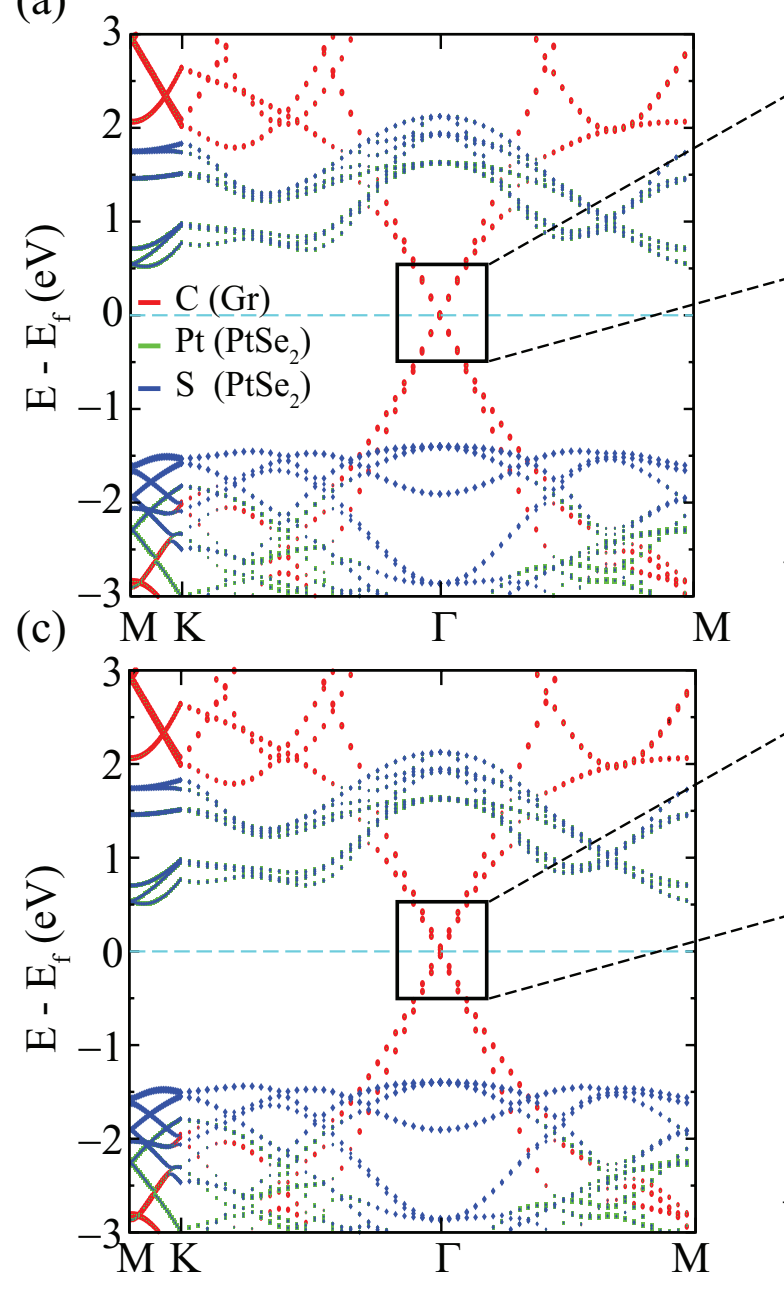

(b)
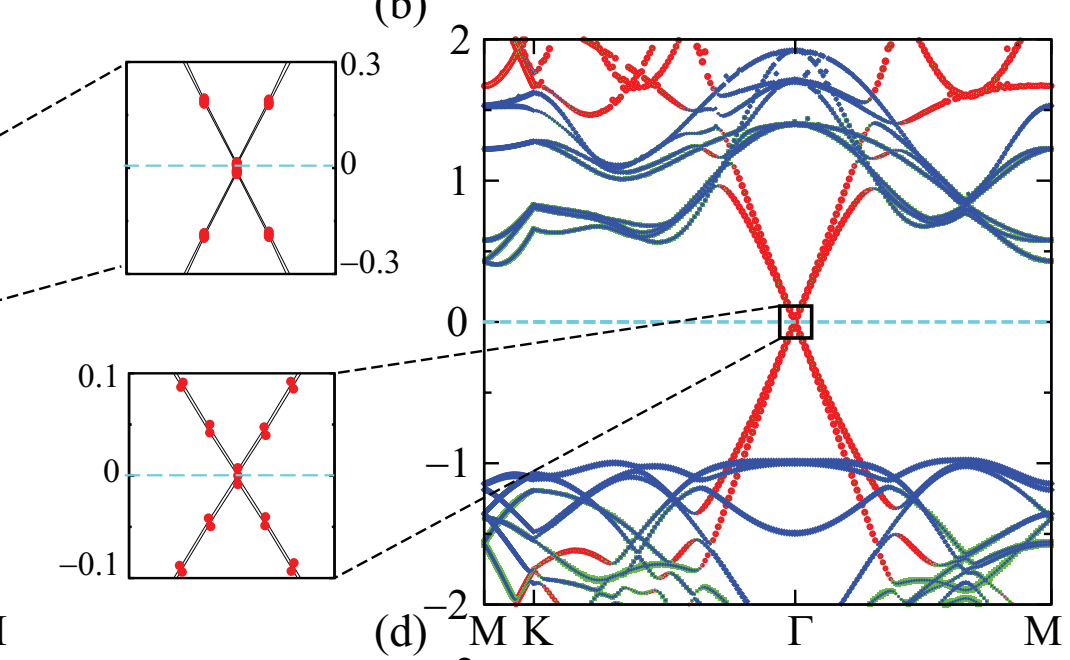

(d) ${ }^{-2} \mathrm{M} \mathrm{K}$
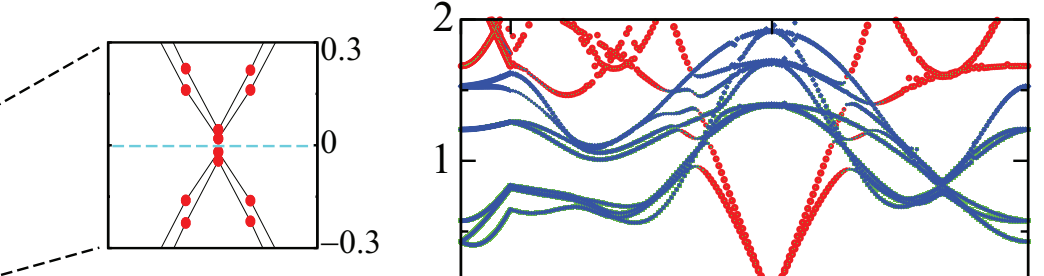

Figure S17: Electronic band structures of (a) stacking 1 and (c) stacking 2 of gr/PtSe ${ }_{2}$ heterostructures under HSE06 calculations, (b) stacking 1 and (d) stacking 2 of gr/PtSe ${ }_{2}$ heterostructures under PBE calculations. 